\title{
STUDY OF STRUCTURAL CHARACTERISTICS OF ANCIENT BRICKS WITH NEUTRON RADIOGRAPHY FACILITY AT BTRR
}

\author{
ROBIN BARMAN $^{1}$, SUdIPTA SAHA ${ }^{1,2}$, MD. SAYED HOSSAIN ${ }^{\varpi, 3}$, ANIK DAS $^{3}$, MD. KAOSAR \\ AHMMAD RABBY ${ }^{3}$, ABDULlAH Al MAHMUD ${ }^{3}$, DEBASISH CHOWDHURY ${ }^{1}$ \\ ${ }^{1}$ Institute of Nuclear Science and Technology, Bangladesh Atomic Energy Commission, Dhaka, 1349, \\ Bangladesh; ${ }^{2}$ Department of Physics, Kyungpook National University, Daegu, 41566, South Korea; ${ }^{3}$ Center \\ for Research Reactor, Bangladesh Atomic Energy Commission, Dhaka, 1349, Bangladesh \\ e-mail: robinapece@gmail.com, sudipta.sust@yahoo.com, sayedrj34@gmail.com, \\ anikbuet83@gmail.com, kaosar167@hotmail.com, almahmudmiad@gmail.com, \\ debasish.chowdhury@baec.gov.bd
}

(Received June 22, 2021; accepted November 28, 2021)

\begin{abstract}
Neutron radiography (NR) has been applied successfully to investigate different types of building materials, rock samples, sculptures, statues or monuments for since long. The utilization of neutron imaging for non-invasive investigations of cultural heritage objects is demonstrated on the example of ancient bricks found in Mahasthangarh and Sonargaon, two key archaeological sites in Bangladesh. The visualization of the internal structure of different brick samples, by means of Neutron Radiography (NR), has been experimented using the BTRR research reactor in Bangladesh - the only neutron imaging facility available in Bangladesh for R \& D purposes. Manufacturing building materials have become a very good option for business in developing countries like Bangladesh. Among the non-destructive testing (NDT) techniques, neutron radiography is the most common procedure to identify light and organic materials, homogeneity, any inclusion or voids or cracks etc. inside the structure. The radiographic images in a dry condition for individual samples have been investigated. The image analysis was performed using ImageJ software and texture features were extracted using gray level co-occurrence matrix implemented by MATLAB for acquiring qualitative and quantitative information from this inspection technique at a high level of accuracy. The results obtained by neutron imaging provide the statement that the brick sample from Mahasthangarh is more homogeneous inside.
\end{abstract}

Keywords: Ancient Brick, Features Extraction, Neutron Radiography, Non-destructive Testing, Research Reactor.

\section{INTRODUCTION}

Cultural heritage artifacts need to be studied by means of non-invasive testing methods due to their delicacy and uniqueness. For this purpose, different methods based on the transmission of radiation, such as neutron, X-ray and gamma imaging are employed and they have proven to be particularly suitable as they provide inside information on the structure and composition of the studied object. The studied objects most often are composite materials and can consist of a large variety of different constituents such as metals, alloys, ceramics, organic (wood (Barman et al., 2021), textiles etc.) and inorganic materials. Besides further substances related to aging (e.g. corrosion products), conservation treatments (e.g. biocides, consolidants etc.) or storage (e.g. moisture) are added to these cultural and historic artifacts. Thus, cultural heritage objects represent a very versatile group with respect to the composition as well as on the questions and topics they represent and need a special technique to study.

Neutron and X-ray imaging are two differing but partially complementary attenuation methods for the investigation of various cultural and historic artifacts. While neutrons have high contrast for hydrogen and thus for organic materials accompanied by good transmittance for most metals, X-rays show high contrasts for metals and good transmittance for materials consisting of light elements. Either technique can be used depending on the composition of the object and the question to be studied (Mannes et al., 2015). A major advantage of neutron imaging over X-rays and other imaging methods like MRI is the high sensitivity to hydrogenous materials or some other light elements and higher penetration depth in metals or ceramics. 
Besides neutron imaging has the distinctive feature of higher contrast between neighboring elements in the periodic table or isotopes of the same element. A large field of applications is utilizing neutron radiation instead of X-rays or both methods in combination. The user community of neutron imaging varies from industrial or materials research, non-destructive testing, geology, archaeology and fundamental research. Imaging with neutron radiation is used worldwide in quality control of explosive devices for mining, defense and industrial applications, for example, to assess oil and water flow in sedimentary rock reservoirs, assessing water damage in aircraft components and the study of hydrogen embrittlement and cracking in zirconium-alloys (Garbe et al., 2017).

English physicist James Chadwick discovered the neutron in 1932. Three years after his discovery, Hartmut Kallmann and Ernst Kuhn initiated neutron imaging work (Chadwick, 1932; Kallmann, 1948). At the end of the Second World War, their work was stopped and little progress was made from their discovery. In the mid-1950's Thewlis utilized a neutron beam with a flux of between $10^{8}$ to $10^{9} \mathrm{n} / \mathrm{cm}^{2}$-s from the BEPO reactor at Harwell (Thewlis, 1956), marking the beginning of the utilization of neutron imaging in practical applications. Neutron radiography emerges as non-destructive testing (NDT) technique with the availability of a higher flux of neutrons from the research reactor and paves the way for insight into a number of challenging practical applications. Neutrons can penetrate high atomic number materials, such as metals, to observe internal low atomic number materials. Neutron imaging begins to change from providing just qualitative insight about an object to a technique that can also provide quantitative information about the details within the object with the improvement of film-based neutron radiography technique. Development of neutron imaging facilities and devices continued from the late 1950's to the present (Brenizer, 2013).

Neutron radiography is a very efficient method to enhance investigations in the field of non-destructive testing. Neutrons have a high sensitivity to hydrous materials of brick's components which are highly visible in the radiographic image compared with other components. Neutron radiography is based on the attenuation of a neutron beam by atomic nuclei. A collimated neutron beam is transmitted through the sample. Neutrons can either be absorbed or scattered, or they can pass undisturbed through the sample. The intensity of the neutron flux after passage through a sample is detected by a two-dimensional neutron sensitive detector screen. Information about the mass and thickness of neutron-attenuating components, such as bricks, can be derived by comparing the transmitted and the unperturbed neutron beam. Digitisation of the radiographs yields two-dimensional gray value images with pixel values proportional to the neutron flux received at the detector screen (Oswald et al., 2008).

The aim of this research is to introduce NR as a non-destructive examination technique and describe the NR equipment at BTRR nuclear reactor that was used for this study. The neutron radiography instrument significantly enhances the research capabilities of the Bangladeshi neutron science facilities at BTRR. It provides university and scientific community-based users of the BTRR research reactor a powerful tool for non-destructive real space testing and evaluation, with properties complementary to $\mathrm{x}$-rays and synchrotron methods. The instrument can be effectively utilized by a large area of scientific research from medical applications, biology and environmental science, geology and engineering science as well as an industrial application which are key areas for future technology and industrial developments in Bangladesh. Neutron radiography finds applications in various fields, such as archaeology, biology, aeronautics, car industry, material studies, etc. The methodology and the application range of neutron imaging techniques have been significantly improved at numerous facilities worldwide in the last decades. We aimed to assess the capabilities of NR combined with image analysis tools for studying non-destructively ancient bricks with particular attention to the quantitative effects of image texture features extracted by means of gray level cooccurrence matrix. The texture is a property that clarifies the structure of an image or is defined as an organized recurrence of pixels or patterns on the exterior. It is complex optical patterns that are collections of pixels, or objects within patterns with the properties of bright effect, colors, shapes, etc.

\section{MATERIALS AND METHODS}

\section{NEUTRON IMAGING FACILITIES}

The design goal for a neutron imaging facility is the bombardment of a specimen with a nearly parallel and properly collimated neutron beam. The neutron beams should have a dimension generally a little larger than the sample and are equally distributed over the field-of-view (FOV) of the detector with well-defined energies. The detector is closely placed behind the sample (Lehmann et al., 2017). The basic experimental set-up of a neutron imaging facility consists of a neutron source, a collimator functioning as a beam 
formatting assembly, a detector and the investigated object, which is placed between the exit of the collimator and the detector and shielding protection from the harmful radiation surrounding workplace. A perfect imaging result is possible only when these individual components are optimized (Zhang et al., 2018).

Neutron imaging activities have been progressed in recent years from a tool for non-destructive testing to more sophisticated research methods for many kinds of investigations and applications (Lehmann and Ridikas, 2015). Neutron imaging is based on measuring the transmitted intensity of neutron beams through an object, either in two dimensions (radiography) or three dimensions (tomography). Currently, more than seventy (70) research facilities perform neutron imaging in over 41 countries. In addition, several new facilities, for example, CARR and CSNS in China, LAHN in Argentina, DINGO at ANSTO in Australia and ODIN at ESS in Sweden, are under development (Zhang et al., 2018). The total number of neutron radiography facilities in different research reactors is depicted in Fig. 1 and Fig. 2 illustrates the geographical distribution of operational research reactors worldwide at present.

Most of the neutron imaging facilities are designed and built in a manner where the beam from an initial source of fast neutrons is extracted towards the sample position after moderation and filtration by a collimator and other beam tuning devices. The transmitted neutron beam is recorded in a 2D image detector which is

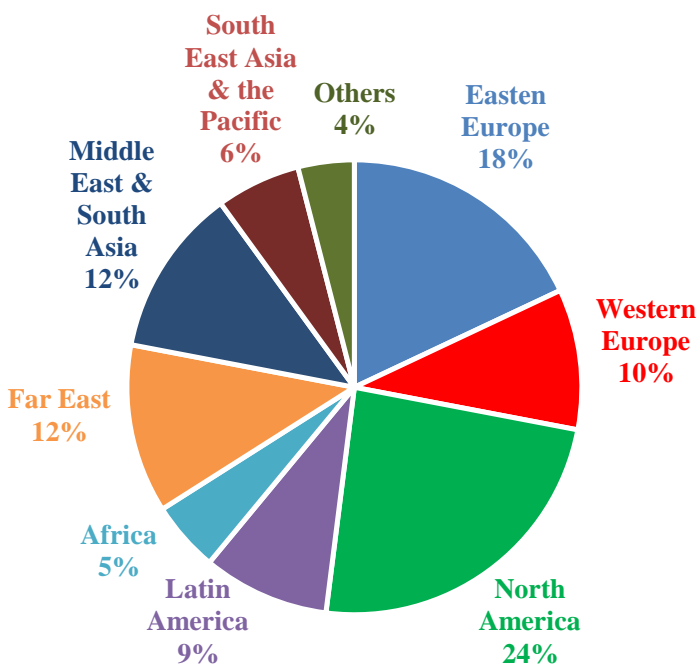

Fig. 1. Regional distribution of Research Reactors based Neutron Radiography Facilities; total number taken from

(https://nucleus.iaea.org/RRDB/Content/Util/NeutronR adioGraphy.aspx) is 74. positioned just behind the sample before the residual beam reaches a beam catcher or a dump. Such facilities are shielded adequately to ensure radiation protection requirements with a controlled access system (Lehmann and Ridikas, 2015).

\section{PRINCIPLE OF TRANSMISSION NEUTRON RADIOGRAPHYTRON IMAGING FACILITIES}

Neutron radiography is the projection of the object in two dimensions. The information is averaged over the thickness of the object along the beam projection path. When neutrons encounter a matter, they are partly absorbed, partly scattered, and partly transmitted. Neutron intensity follows the exponential attenuation law of Beer-Lambert. The intensity of the transmitted beam, $I\left(\mathrm{n} / \mathrm{cm}^{2}\right.$-s), i.e. the number of particles passing across a unit area in unit time, can be described as follows:

$$
\mathrm{I}=\mathrm{I}_{0} \mathrm{e}^{-\Sigma \cdot \mathrm{x}}
$$

where, $\mathrm{I} 0\left(\mathrm{n} / \mathrm{cm}^{2}-\mathrm{s}\right)$ is the intensity of the neutron beam leaving the collimator and penetrating the object, $x$ $(\mathrm{cm})$ is the total thickness of the object along with the beam projection and $\Sigma\left(\mathrm{cm}^{-1}\right)$ is the neutron linear attenuation coefficient or the macroscopic crosssection. For a substance containing more than one element, the total macroscopic cross-section is determined by the addition of its constituent elements (Zhang et al., 2018).

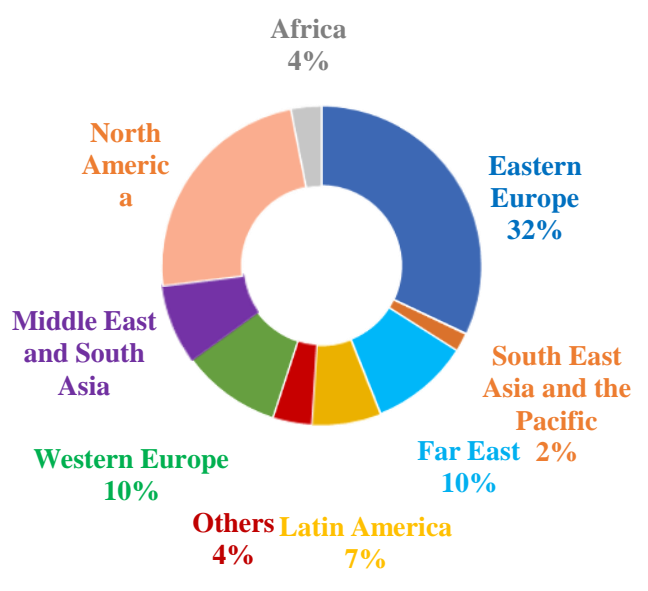

Fig. 2. Distribution of operational research reactors around the world; the total number taken from (https://nucleus.iaea.org/RRDB/Content/-Stat/StatO.aspx) is 223, status 2021. 
This technique is based on the absorption and scattering of a neutron beam as it passes through a sample. With this technique, the inner macroscopic structure and material composition of the sample can be visualised (Orina et al., 2017).

Neutron radiography (NR) has been demonstrated by several researchers to be one of the NDT methods for over 40 years. The best image quality NR can be obtained by using $\mathrm{Gd}$ as the neutron converter screen and X-ray film as the recording medium but high intensity neutrons are needed due to its slow speed. It is, therefore, practical to carry out NR with neutron beam from a research reactor but not from other neutron sources like neutron generators and Cf-252 (Chankow et al., 2010). Depending on the availability of the converter materials, film based neutron radiography experiments are conducted in two ways namely, direct film method and indirect film method as shown in Fig. 3. In the direct film method, the film is placed in close contact with the converter material and the converter materials do not become radioactive but do emit radiation promptly after absorption of the neutrons during experiments. Gadolinium, boron, lithium and cadmium are used as converter materials in the direct exposure method. This technique is fast but prone to background radiation effects.

In the indirect or transfer method, the film is not placed with the converter material during exposure rather only the converter is exposed to neutron radiation. The converter materials become radioactive after neutron absorption. The film is then placed with the converter in a separate area and leaves the converter to decay. Since the film is not exposed to neutron directly it takes more time than the direct method. Indium, dysprosium, gold and samarium are mainly used in the indirect method. This method is suitable where the background radiation level is very high since it is less prone to background radiation.

Neutron radiography has the capability to reveal detailed components that are not visible in an x-ray image. Neutrons can penetrate many heavy materials such as titanium and lead. This allows for some unique applications, which is impossible with $\mathrm{X}$-ray or Gamma-ray radiography. Thus, neutron radiography supplies a unique contrast mechanism not found with other imaging methods based on electrons, X-rays, or nuclear magnetic resonance. Elements having adjacent atomic numbers can have widely different absorptions of neutrons and it varies from element to element, even from isotope to isotope. Neutrons also provide high quality radiographs of highly radioactive components. The contrast mechanism can be established for a neutron radiography image by the darker area that represents the lower attenuation and the lighter area representing the greater attenuation. Neutron radiography images can be produced with contrast set by different attenuation due to different elements (Luo, 2007).

\section{GLCM \\ TEXTURE FEATURE EXTRACTION USING}

Texture analysis is used in a very broad range of fields and applications, from texture classification such as remote sensing, biometric issues, medical images and pattern recognition for image retrieval. For each of these image processing operations, it is needful to extract significant texture features, that can be obtained by Gray Level Co-occurrence Matrix (GLCM). Gray level co-occurrence matrix (GLCM) is a popular texture-based feature extraction method. In image analysis, texture feature is the result from the observed groups of the intensity in specific locations statistical distribution relative to each other from the image. The GLCM is a matrix of how often times several sets of a pixel in grey levels exist in an image. GLCM is computed for a selected pair of distance and angle. For a specific pair of distance and angle relative recurrences are computed of that pair of each pixel and its neighboring as shown in Fig.4 (Alazawi et al., 2019).

The GLCM determines the textural relationship between pixels by performing an operation according to the second-order statistics in the images. Usually, two pixels are used for this operation. The GLCM properties of an image are expressed as a matrix with the same number of rows and columns as the gray values in the image. The elements of this matrix depend on the frequency of the two specified pixels. Both pixel pairs can 

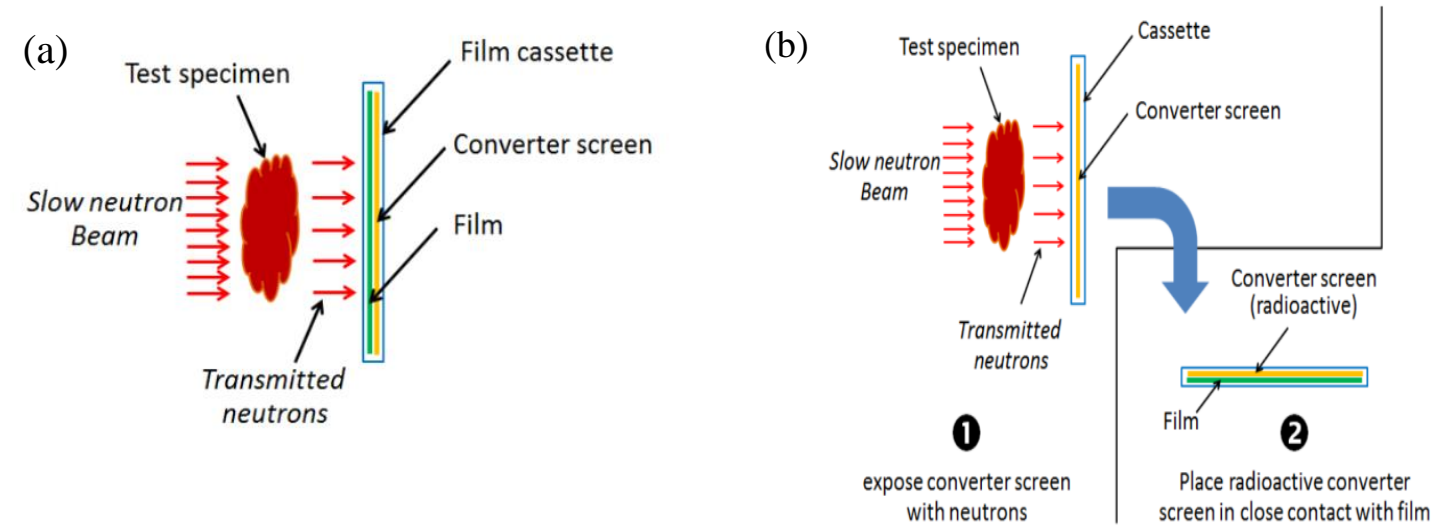

Fig. 3. Illustration of (a) direct exposure method; (b) two steps of indirect or transfer exposure method

vary depending on their neighborhood. These matrix elements contain the second-order statistical probability values depending on the gray value of the rows and columns (Öztürk and Akdemir, 2018).

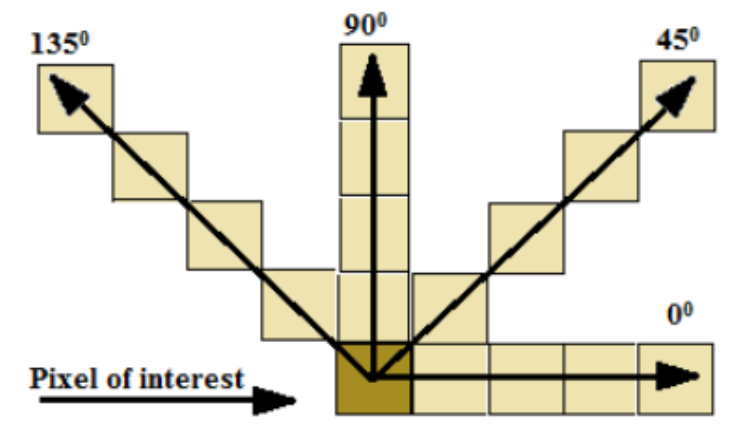

Fig. 4. Angle relative of interesting pixel and its neighboring.

\section{SAMPLE COLLECTION AND HISTORIC IMPORTANCE OF THE SITES}

Historical places are very important for any country to represents its culture, traditions and religions. It preserves the traditions and cultural identity of any country with them which can be a great source of knowledge for future generations (Islam and Kabir, 2019). Mahasthangarh and its environs are a potential heritage site and one of the prominent earliest fort city centers of South Asia that help to understand chronological history along with the cultural development of ancient civilizations of Bengal Delta. The Mahasthangarh is situated at the Shibgonj thana of Bogura district, the northwestern part of Bangladesh. Alexander Cunningham was the first European scholar who brilliantly identified the ruins of Mahasthan as the capital city of Pundravardhana in 1879 (Khan, 2020).
The geographical location of the Mahasthangarh is shown in Fig. 5 (a).

(https://en.banglapedia.org/index.php?title=Mahasthan)

A large number of significant movable and immovable artifacts have been discovered from the Mahasthangar. The major findings are Brahmi inscription dated back 3rd century BCE, Arabic inscription dated 13th century CE, Aurangzeb's Sanad (decree of Muslim ruler) and Parsi inscription dated $1718 \mathrm{CE}$, which indicate the rich history of Mahasthangarh. The site's existing ruins and archaeological findings (artifacts) elucidate the transformation of political, social, cultural, economic, religious, and artistic values from the early historic (4th century BCE) to the colonial period (19th century CE) (Khan, 2020).

As the capital of the fifteenth-century Bengal ruler Isa Khan, and once an important trading and political center, Sonargaon boasts architecture of the Sultanate, Mughal, and colonial periods. The Sonargaon historic city, located near the present-day capital of Dhaka, Bangladesh, includes several Mughal monuments. Old Sonargaon can be found near Narayanganj and Dhaka, cities in Bangladesh, and is considered one of the first and oldest capitals of Bengal. Sonargaon was known as "The City of Panam" and was a focal part of the renowned Deva Dynasty until the thirteenth century (Sharmin, 2019). The geographical location of the ancient trade city Sonargaon is given in Fig. 5 (b) (https://earth.google.com/web/search/Sonargaon/@ 23.6431314,90.6020637,6.85087328a,10647.842304 8d,35y,0h,0t,0r/data=CigiJgokCQTqpEnszdAEUemOC ukDdAGfmgVVdJqlZAITu4XtVymlZA). 

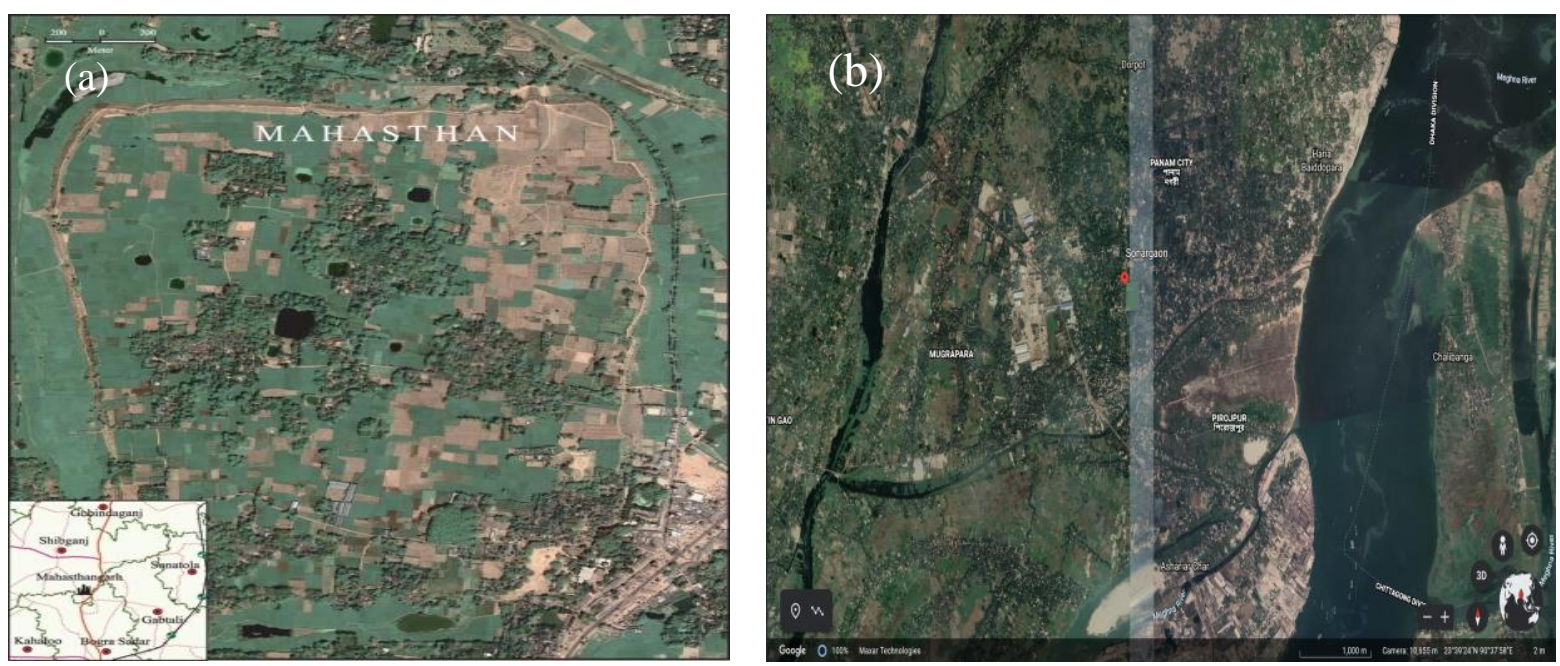

Fig. 5. (a) Satellite image of Mahasthangarh (Banglapedia, 2015); (b) Google earth image of Sonargaon and its surroundings).

Two samples were collected from Mahasthangarh, Bogra which is an ancient city, discovered in 1931, dates to at least 3rd century BC (Hossain, 2006) and the fortified area was in use until the 8th century $\mathrm{CE}$ (Department of Archaeology, Ministry of Cultural Affairs, 2003) and lies between latitudes $24^{\circ} 50^{\prime} \mathrm{N}$ and $25^{\circ} 0^{\prime} \mathrm{N}$ and longitudes $89^{\circ} 15^{\prime} \mathrm{E}$ and $89^{\circ} 30^{\prime} \mathrm{E}$ (https://en.banglape-

dia.org/index.php?title=Mahasthangarh,_Physica-1_Setup). The other two samples were collected from Sonargaon, Naraynganj, Dhaka which was inaugurated as capital during the Buddhist reigning period in the middle of 13 th century and lies between $23^{\circ} 37^{\prime} 09^{\prime \prime} \mathrm{N}$ and $90^{\circ} 35^{\prime} 59^{\prime \prime} \mathrm{E}$

(https://en.banglapedia.org/index.php?title=Mahasthan

garh,_Physical_Setup). The samples, collected for the present study are nearly 150 years old.

\section{THERMAL NEUTRON RADIOGRAPHY FACILITY AT BTRR}

Reactors are excellent sources of thermal neutrons. The neutron radiography for this study were obtained at the BTRR. BAEC TRIGA Research Reactor (BTRR) is the only research reactor of the country located in Savar, at a distance of $40 \mathrm{~km}$ from Dhaka and operated by the Center for Research Reactor, Atomic Energy Research Establishment (Das et al., 2021a,b). The TRIGA MK-II research reactor is a pool-type; light water-cooled and zirconium-hydride moderated reactor as shown in Fig. 6 (a).

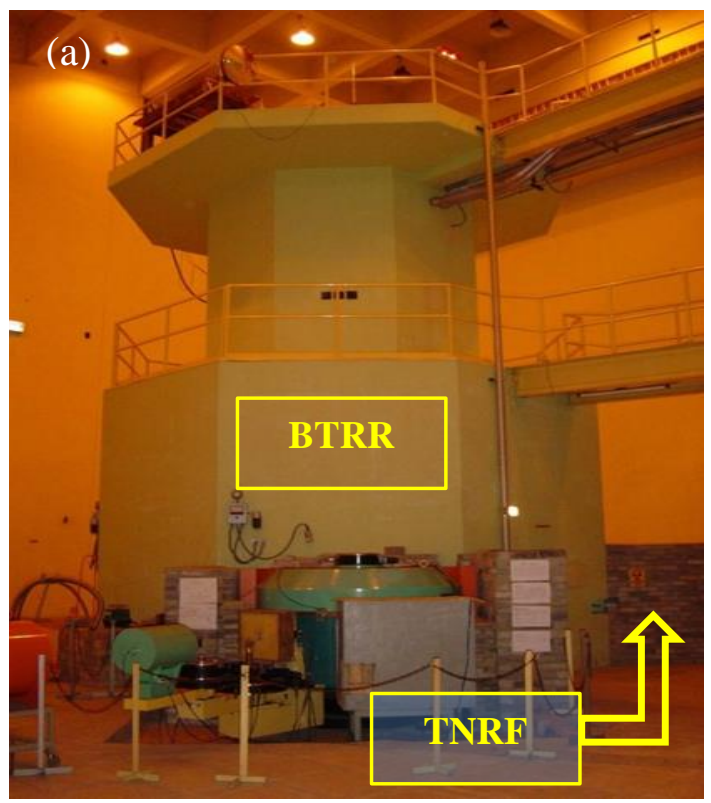

(b)

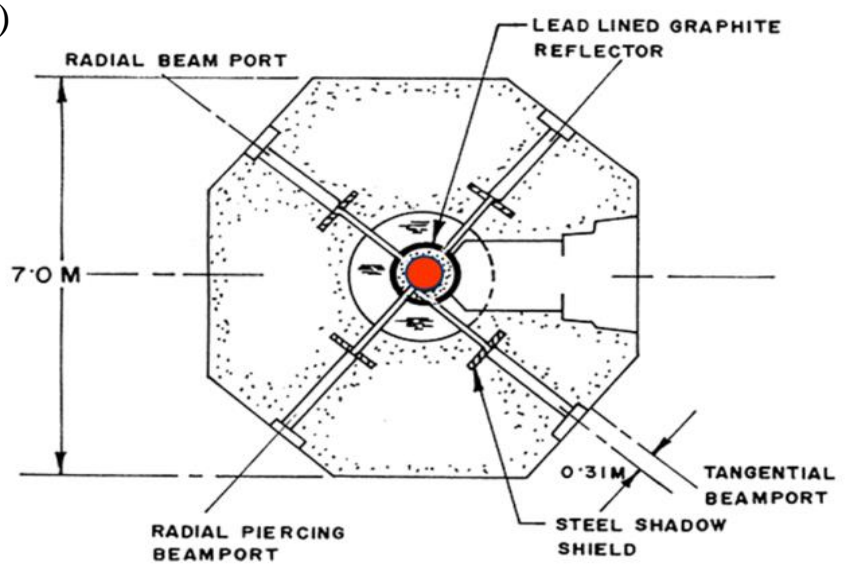



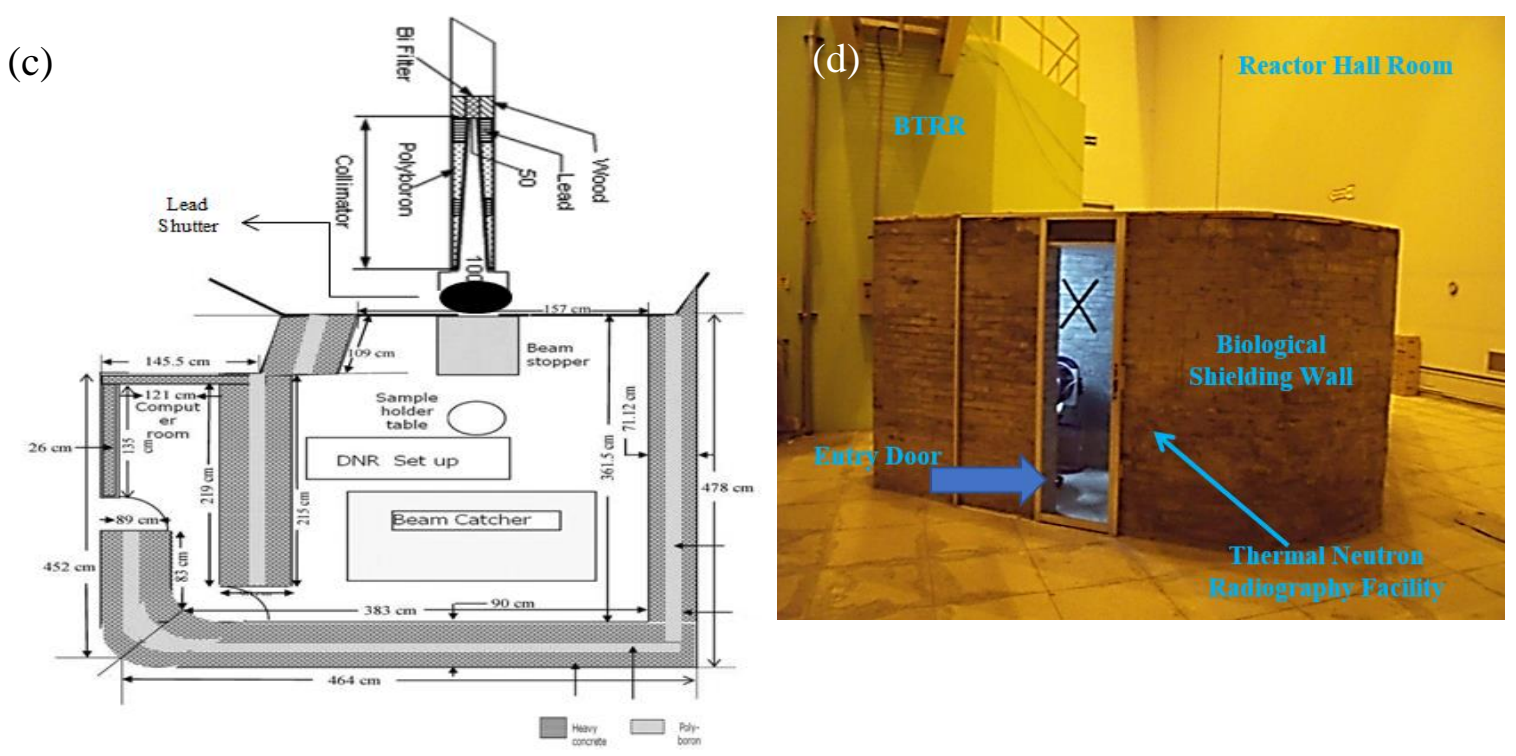

Fig. 6. (a) 3MW BAEC TRIGA Mark-II Research Reactor; (b) Different beam port facilities at BTRR; (c) Schematic diagram of the thermal neutron radiography facility; (d) Biological shielding house of TNRF at BTRR.

Thermal Neutron Radiography Facility (TNRF) has been installed at the tangential beam port of the $3 \mathrm{MW}$ BAEC TRIGA Research Reactor (BTRR). The tangential beam port has been used for neutron radiography facility because of its less amount of gamma rays emission compared to other three beam ports (two Radial Beam Ports and one Radial Piercing beam port) as shown in Fig. 6 (b) and also because of sufficient thermal neutron flux. The quality of neutron radiographs using this facility has been found to be category-I. The NR facility consists of a Bi-filter, a cylindrical divergent collimator, a beam stopper, a sample and camera holder table, a beam catcher and a semi-building biological shielding house. The full schematic illustration of these facilities is provided in Fig. 6 (c). The Fig. 6(d) is depicting the materialistic structure of biological shielding of the entire neutron radiography facility.

\section{EXPERIMENTAL SETUP}

Loading the Converter Foil and Detector in the NR Camera. The object being imaged is located in front of a neutron radiography (NR) camera system containing a film and metal converter foil. In the NR camera, gadolinium foil of $25 \mu \mathrm{m}$ thickness was used as a converter and Agfa structurix D4 DW industrial Xray films were used as a detector in the NR experiments. During loading the film and converter foil in the NR camera, the emulsion surface of the film was kept in contact with the converter foil. The camera was then closed tightly. All these procedures have been done in the darkroom.
Setting the NR camera and sample in the neutron beam. The experiments were carried out at the end position of the BTRR tangential beamline using an NR cassette system with conventional $x$-ray film as a recording medium and $\mathrm{Gd}$ metal foil as a converter. The sample and the NR camera were placed on their respective tables across the neutron beam. To ensure a stable positioning of the bricks during the radiography the brick sample was attached to the surface of the NR camera and fixed with aluminium thread tape.

Irradiation condition. Four types of brick samples were studied by using thermal neutron beams from BTRR. To take neutron radiographs samples were irradiated by opening neutron beam at $2.4 \mathrm{MW}$ reactor power level. The direct film neutron radiography method was utilised to capture the inner details of the samples as shown in Fig. 7 with different exposure times. After irradiation, the irradiated film is separated from the NR camera in the darkroom.

Transferring the NR camera. The irradiated film i.e. NR camera is not allowed to take outside the reactor hall till the radioactivity drops down to the permissible level. Then the NR camera was transferred to a dark room with a polyethylene bag by using a tong (1 meter long). The darkroom facility has been prepared for developing neutron radiographs. Each irradiated film was chemically processed with Kodak D19 developer powder and Kodak fixer powder to visualize the sample image.

Strength of the irradiated samples. The radioactivity strength of the sample depends on the intensity of the neutron beam, compositions, density, thickness and 
neutron cross-section of the sample. The samples were irradiated with a thermal neutron flux of $\sim 10^{4} \mathrm{n} / \mathrm{cm}^{2} / \mathrm{s}$. In most of the cases the radioactivity of the sample becomes slightly higher than the background level.

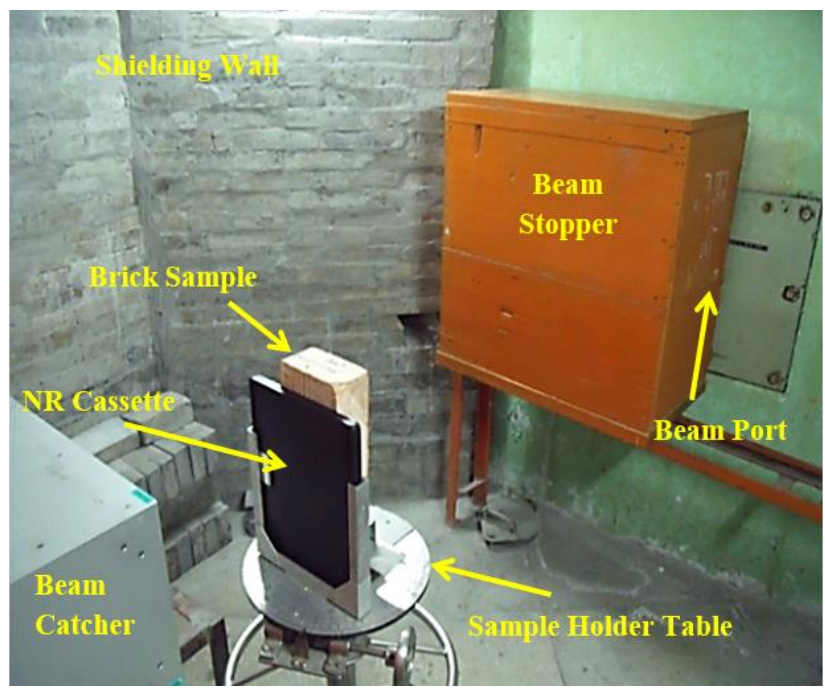

Fig. 7. Experimental setup of direct film NR method.

Data processing and evaluation. Evaluation and visualization of the data sets from 2-D radiographs were carried out using a Java-based image processing program named ImageJ developed at the National Institutes of Health and the Laboratory for Optical and Computational Instrumentation

(https://imagej.nih.gov/ij/download.html). Further data acquisition was performed by using the MATLAB image processing module.

The developed images were digitised with a digital camera. The ImageJ software was used to get 8-bit image from RGB image of a camera. This image processing technology provides the capability to acquire, enhance and analyse the images. Different techniques, such as filtering, edge detection and pseudo-coloring are applied routinely to improve the visualization of the radiographic image. Finally, MATLAB code was run to extract some common texture features of the images using the concept of gray level co-occurrence matrix.

\section{RESULTS AND DISCUSSION}

Direct film NR technique had been applied in this experiment to have the images of the internal structure of the brick samples and observe their characteristics. Two brick samples collected from Mahasthangarh are coded as MB-1 and MB-2 and those collected from Sonargaon are named as SB-1 and SB-2. The radiographs had been obtained after optimum exposure of thermal neutron beam for 3.5 minutes for samples SB-1, SB-2 and MB-1 and 5 minutes for sample MB-2 (top and lateral view) respectively.

The brick sample MB-1, radiograph, region of interest (ROI), edges and the corresponding gray values and surface diagram had been depicted in Fig. 8 and Fig. 9. In Fig. 8 (c) the ROI was selected in the middle of the sample to ensure it covered most of the area of the sample. The original radiograph image was converted to 8-bit image from RGB image. The roughness of the surface of the sample MB-1 was visualized in Fig. 8 (d).

Variations of gray values with pixels positions in 2-D and 3-D were depicted in Fig. 9 (a) and Fig. 9 (b) respectively. It was clearly observed from these two figures that the gray values dipped in the middle region of the sample. This behavior may be attributed to the internal roughness and some porosities in the middle region of the sample. The gray values are distributed from 0 to 255 in Fig. 9 (a) and (b) since these figures represented the 8-bit image of the sample. 0 means a dark area while 255 indicates a brighter region.

As we moved from the central region to the edges the gray values gradually increased. The maximum gray value was observed as 234.1519 at a pixel position of 2 . The gray gradually decreased as we moved to the next pixel location. Minimum gray value was found at pixel location of 133 and the value was 196.2386. The variation of the gray values in the ROI was $16.19 \%$ for sample MB-1. Fig. 9 (a) only showed the gray value distribution of ROI while Fig. 9 (b) showed the gray value distribution of the whole region including the background. The outer dark area of the central dome in Fig. 9 (b) represented the background information of the 8-bit image. 

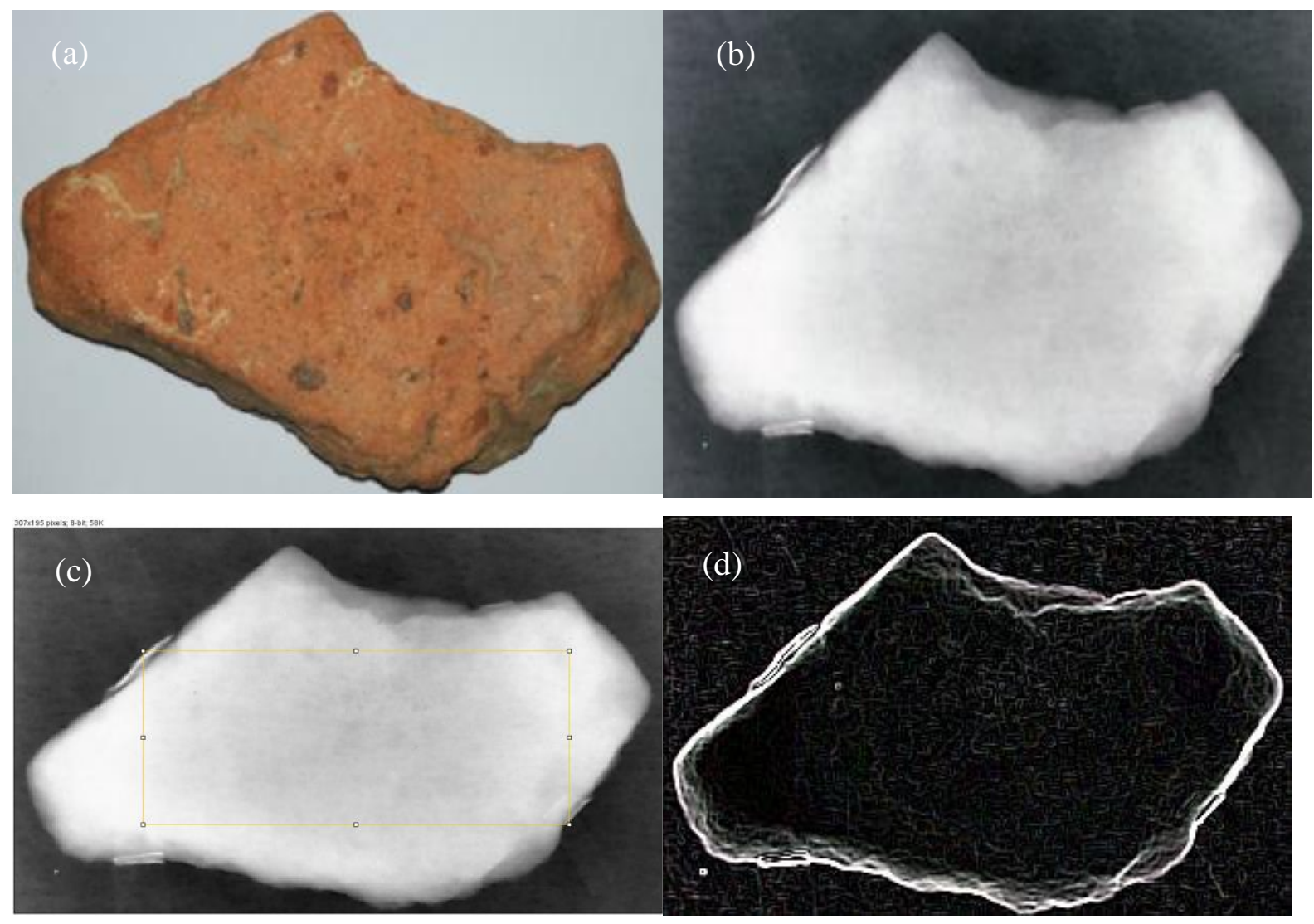

Fig. 8. (a) Mahasthangarh Brick Sample (MB-1); (b) 2D transmission radiograph of MB-1(307 x 195 pixels; RGB; 234K); (c) ROI of 8-bit image from RGB image of MB-1(8-bit; 58K); (d) Edge detection of MB-1.

(a)

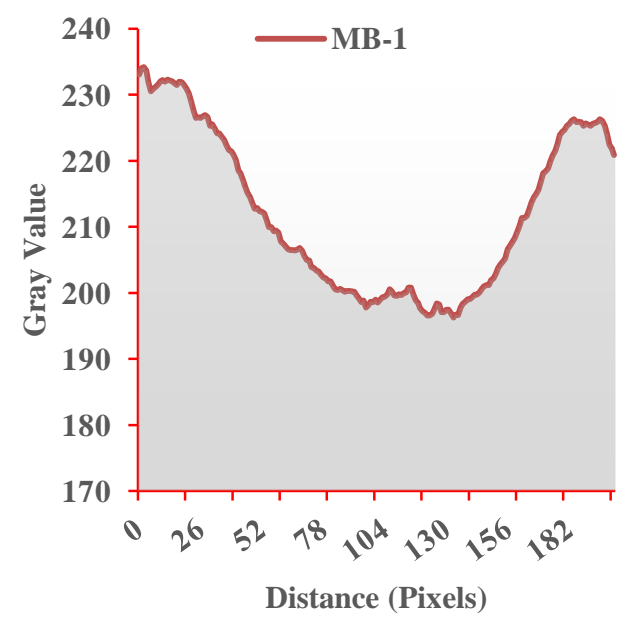

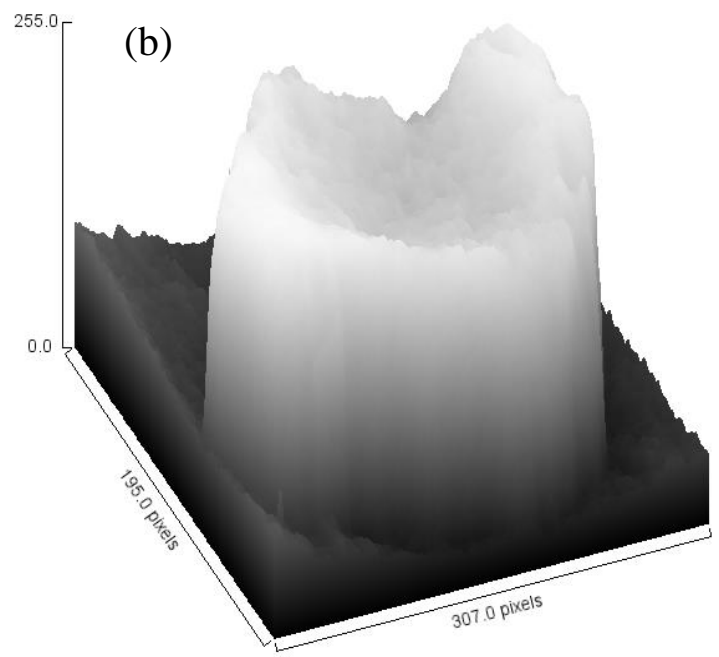

Fig. 9 (a) Gray value distribution of MB-1 at different pixel positions for ROI; (b) 3-D Surface plot of 8-bit image of $M B-1$

The top view of brick sample MB-2 and its radiographs, ROI and edges were illustrated in Fig. 10. The edges and surface roughness were clearly visualized in Fig. 10 (d). The gray values distribution in two dimensions with pixel locations for ROI was depicted in Fig. 11 (a) while Fig. 11 (b) showed the distribution of gray values in 3-D along with the background. 

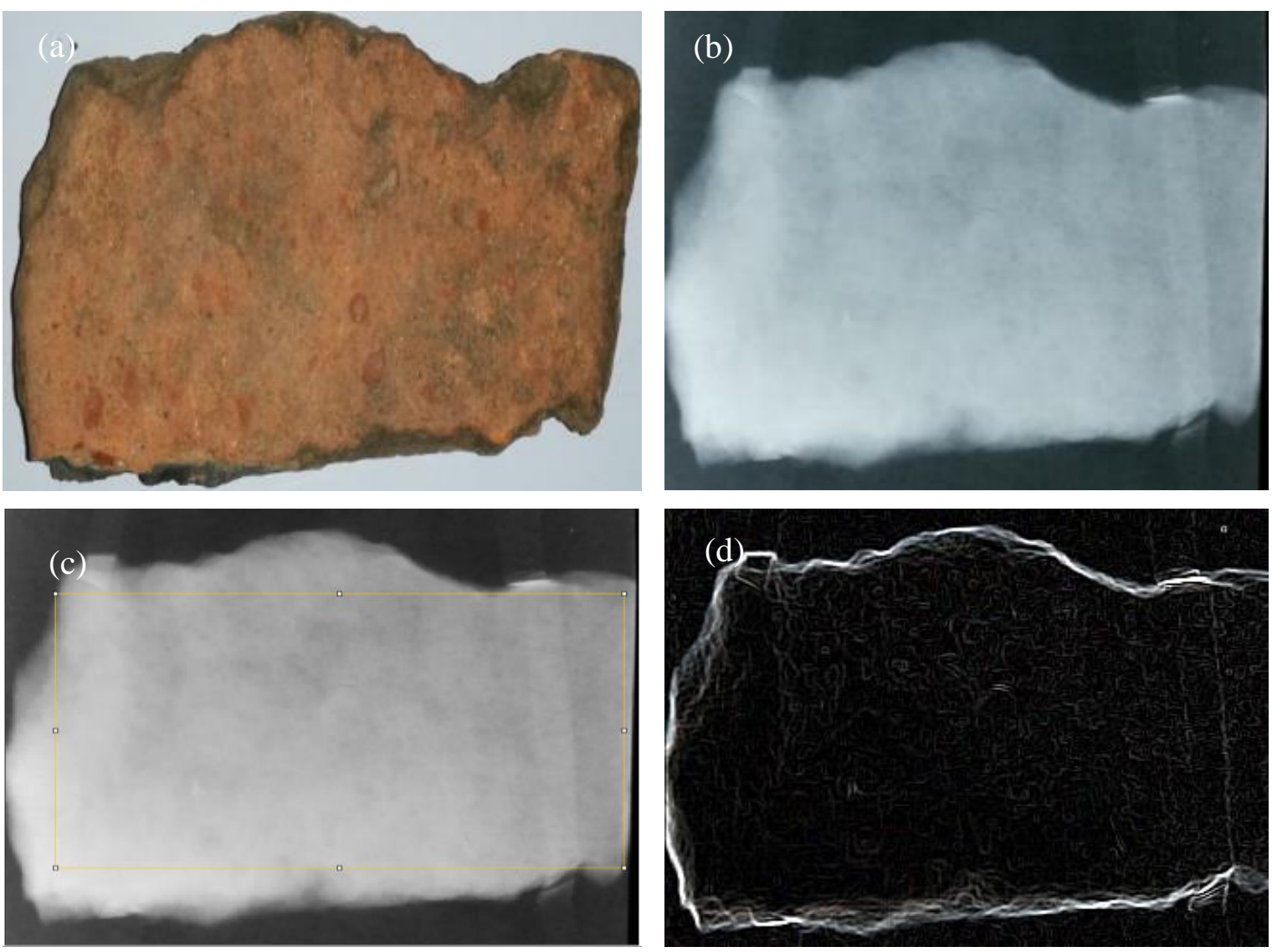

Fig. 10. (a) Top view of Mahasthangarh Brick Sample (MB-2); (b) RGB radiograph of MB-2 (327 x 260 pixels; $R G B ; 332 \mathrm{~K})$; (c) ROI as 8-bit image extracted from RGB image of MB-2 (8-bit; 83K); (d) Edge detection of MB-2 (top view).
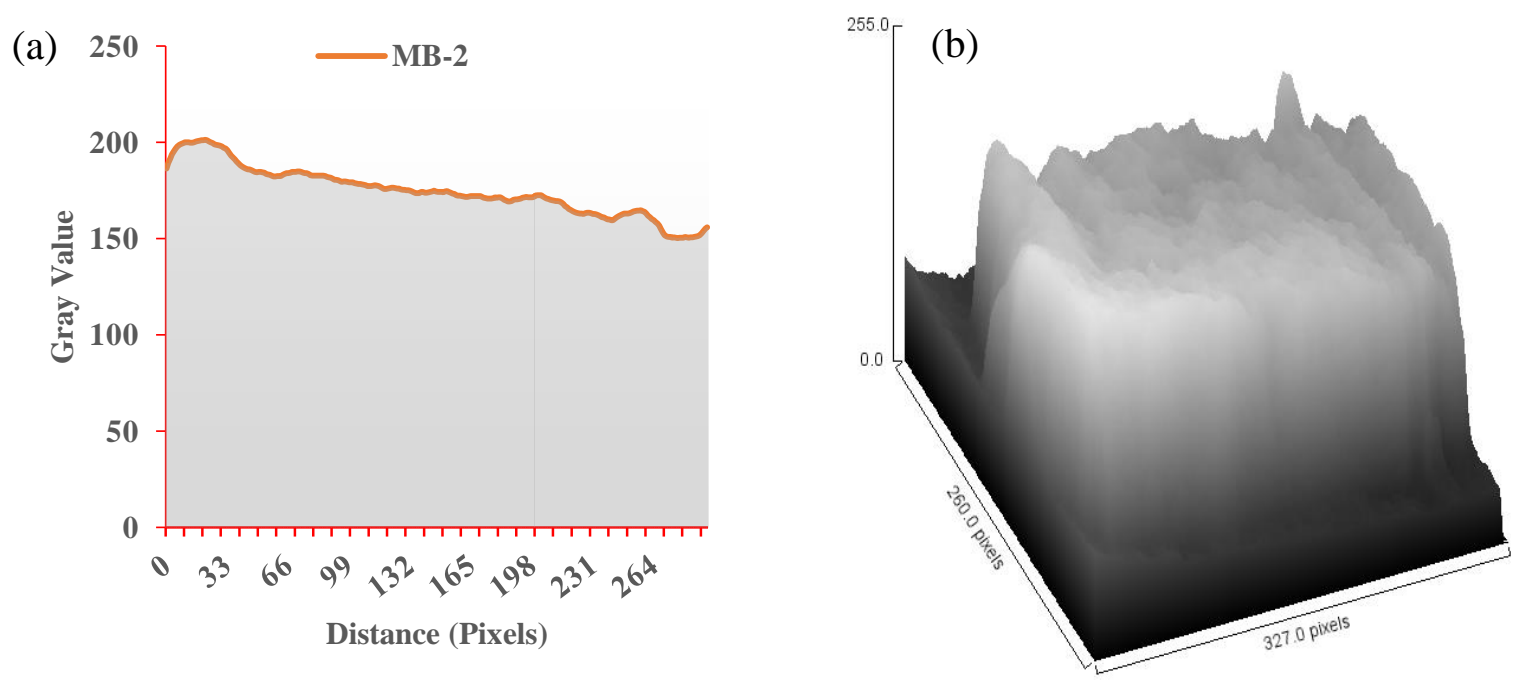

Fig. 11. (a) Gray value of MB-2 (top view) for ROI at different pixel positions; (b) Surface plot of 8-bit image of $M B-2$ (top view).

The gray values changed throughout the ROI. At one end of the ROI, the intensity level was slightly lower than the other extreme end of ROI. No drastic change was observed in gray values across ROI. But the gray values were decreasing with increasing pixel positions. The highest value of the intensity was found at 201.1553 at pixel position 21 . The lowest value was observed as 150.2547 at a pixel location of 277 . The variation in gray value was $25.30 \%$ for sample MB-2 (top view). It meant there were fewer voids and porosities in the ROI of the sample since there was no 
drastic dip of intensities in the central region. But large variation in gray values indicated that there were erosions and deformations at the edges of the sample.

The lateral view of brick sample MB-2 was shown in Fig. 12 (a). The radiograph, ROI and edge formation were depicted in Fig. 12 (a) to (c). The intensity distribution in 2-D and 3-D format was given in Fig. 13 (a) and (b). From Fig. 14 (a) it was seen that the gray value distribution curve resembled a parabolic shape. The maximum gray value was 183.2751 which occurred at the 37-pixel position and the minimum gray value was 141.455 at a pixel location of 77 . Variation in gray value intensity was $22.82 \%$. The lateral view of the MB-2 sample provided a flat curve representing no layer deformation inside the sample.
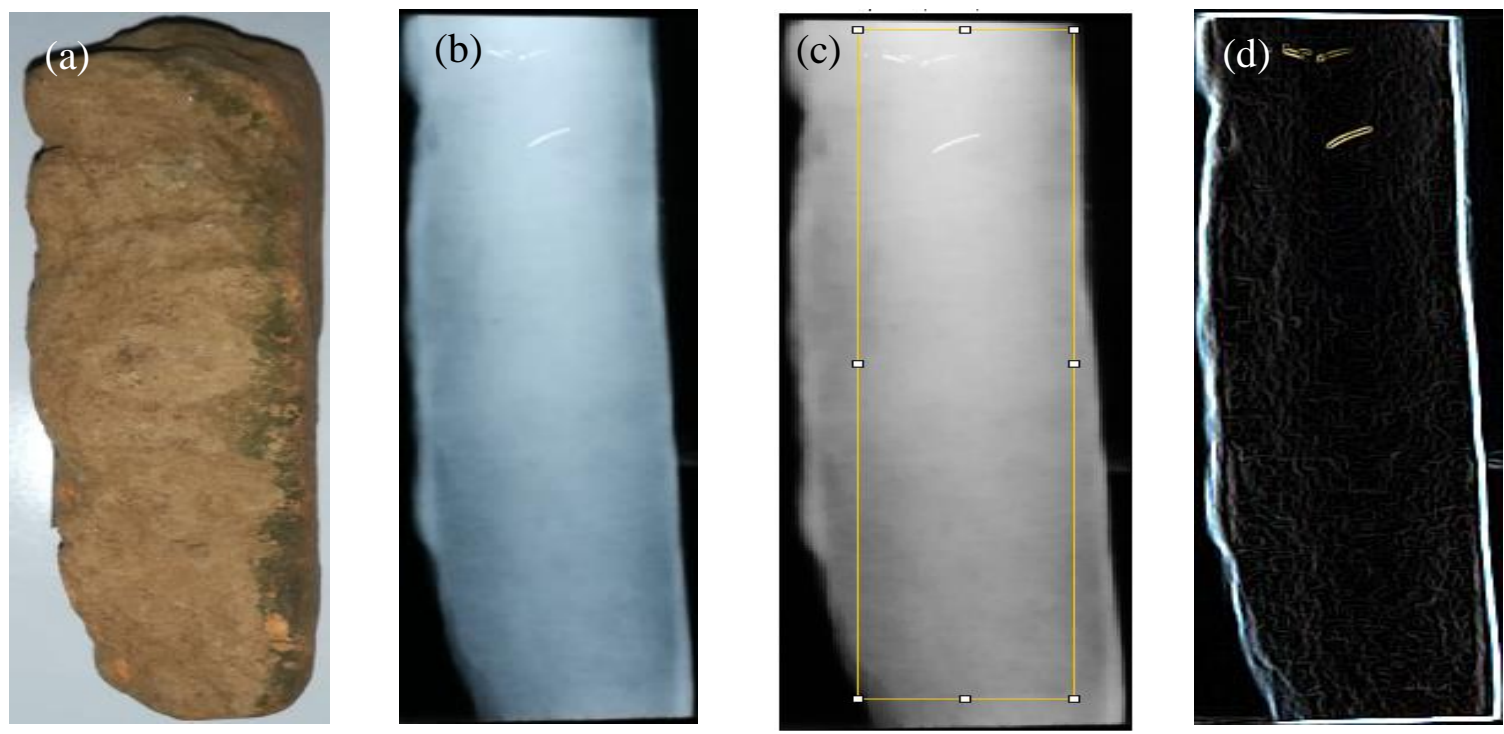

Fig. 12. (a) Lateral view of MB-2; (b) Lateral radiograph of MB-2 (126 x 416 pixels; RGB; 205K); (c) ROI of MB-2 (lateral view) (8-bit; $51 \mathrm{~K})$; (d) Edge finding of $\mathrm{MB}$-2 (lateral view).
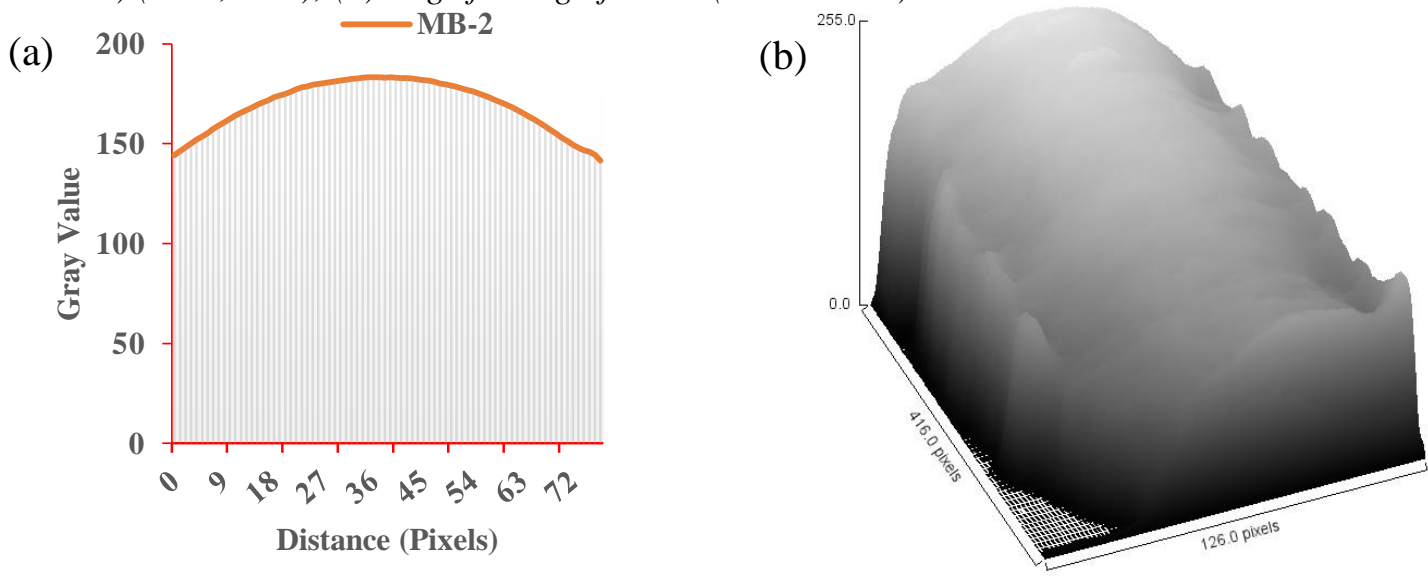

Fig. 13. (a) Variation of gray value with pixel position for ROI of MB-2 (lateral view); (b) 3-D surface plot of 8-bit image of $M B-2$ (lateral view).

The brick sample from Sonargaon (SB-1), a radiographic image of the sample, ROI of the $\mathrm{SB}-1$ and edge detection were shown and depicted in Fig. 14. A shadow dark spot was visible in the ROI of the radiograph which implied the porous surface of the brick sample. Besides the edges were prominent in Fig. 14 (d) which indicated that SB-1 had a very rough and eroded surface. 
(a)

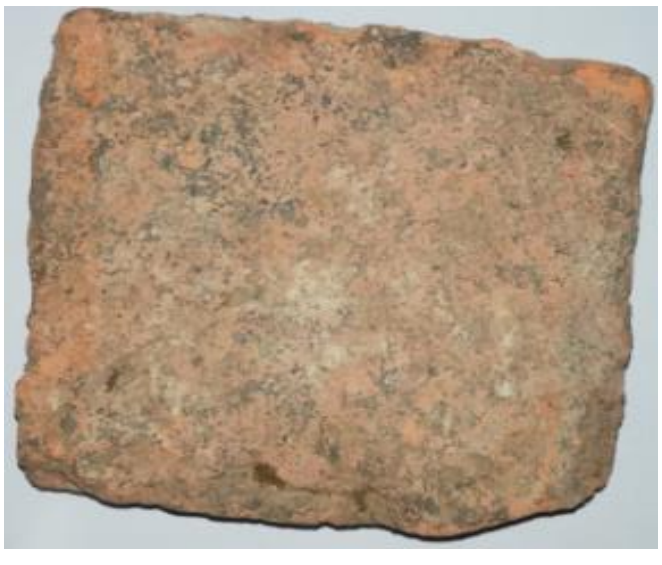

(c)

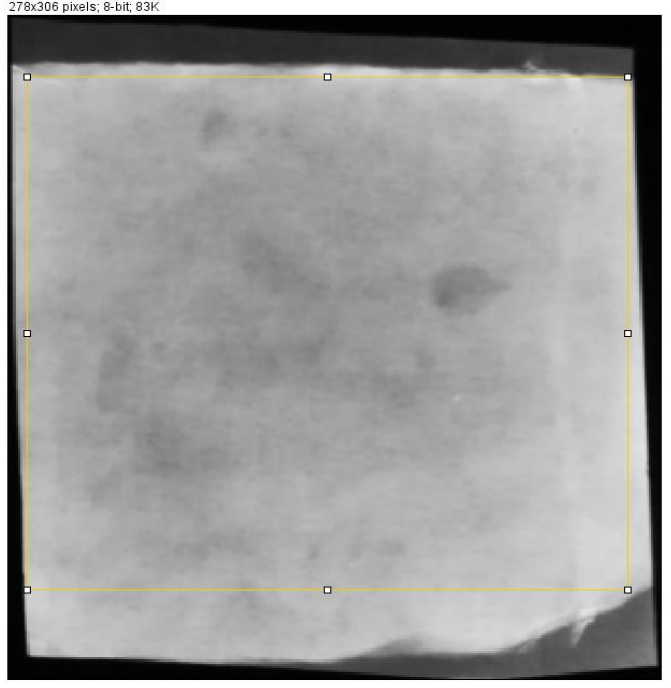

(b)

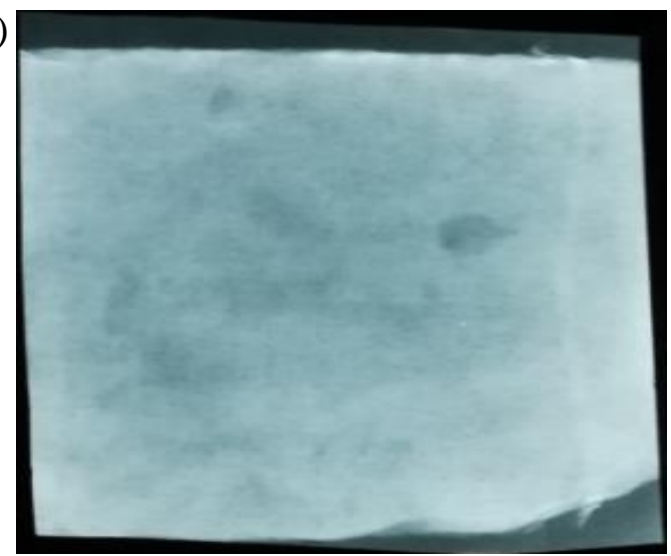

(d)

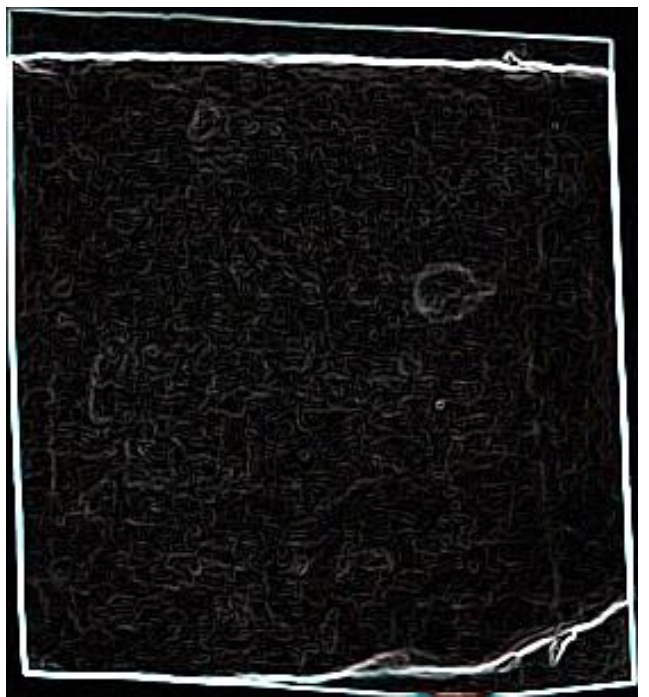

Fig. 14. (a) Brick sample from Sonargaon (SB-1); (b) Radiograph of SB-1(278 x 306 pixels; RGB; 332K); (c) 8-bit image and ROI of SB-1 (278x306; 8-bit; 83K); (d) Detection of edge of SB-1.

(a)

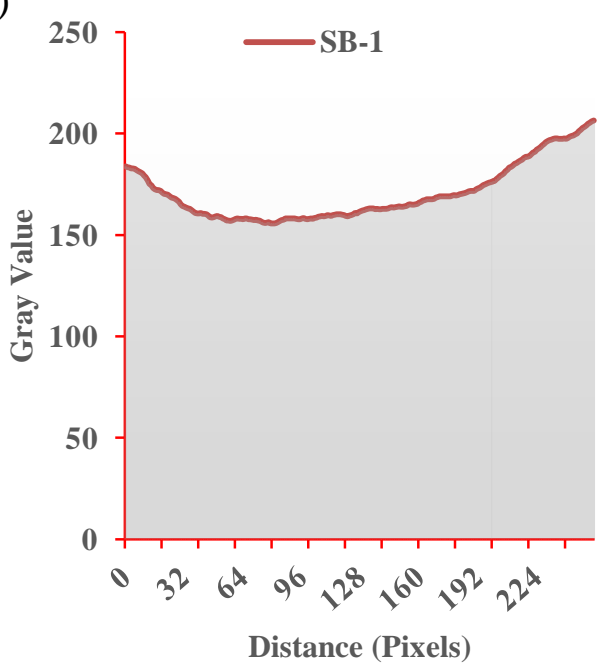

(b)

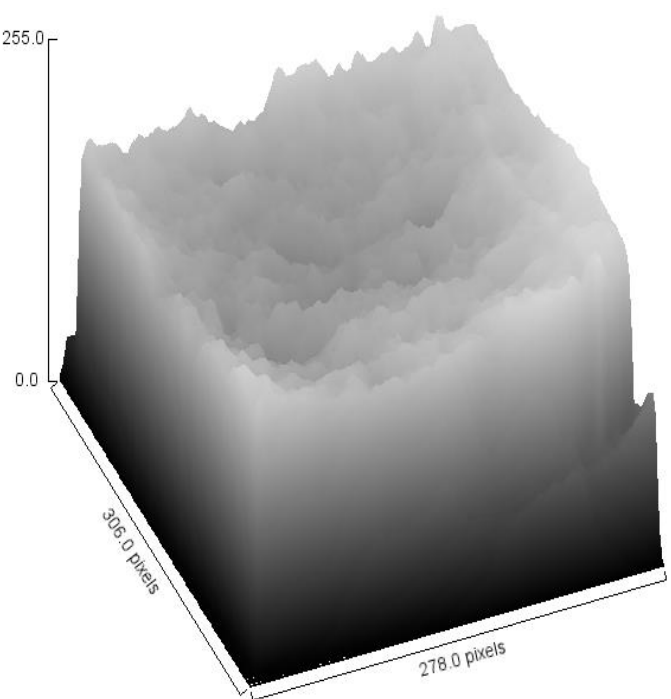

Fig. 15. (a) Image intensity at different pixel distances for ROI of SB-1; (b) Gray value distribution in 3-D surface plot of 8-bit image from $S B-1$.

The intensity variation of the SB-1 sample was depicted in Fig. 15. From these gray value distribution curves, it was seen that intensity gradually increased from the left side of the graph (Fig. 15 (a)) to the right 
side. SB-1 image had a maximum intensity of 206.4726 at pixel position of 255 and minimum intensity of 155.6287 at pixel location of 80 . The variation from maximum to the minimum value of intensity was about
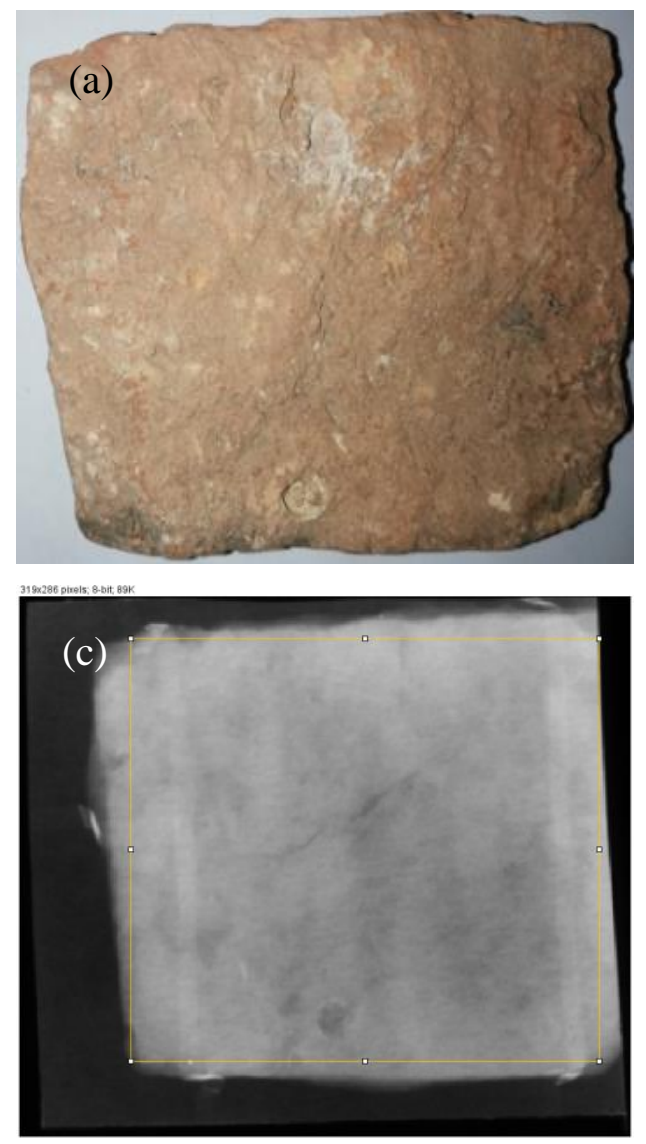

24.63\%. The surface plot in Fig. 15 (b) showed a slight depression in intensity value towards one end of the intensity profile.
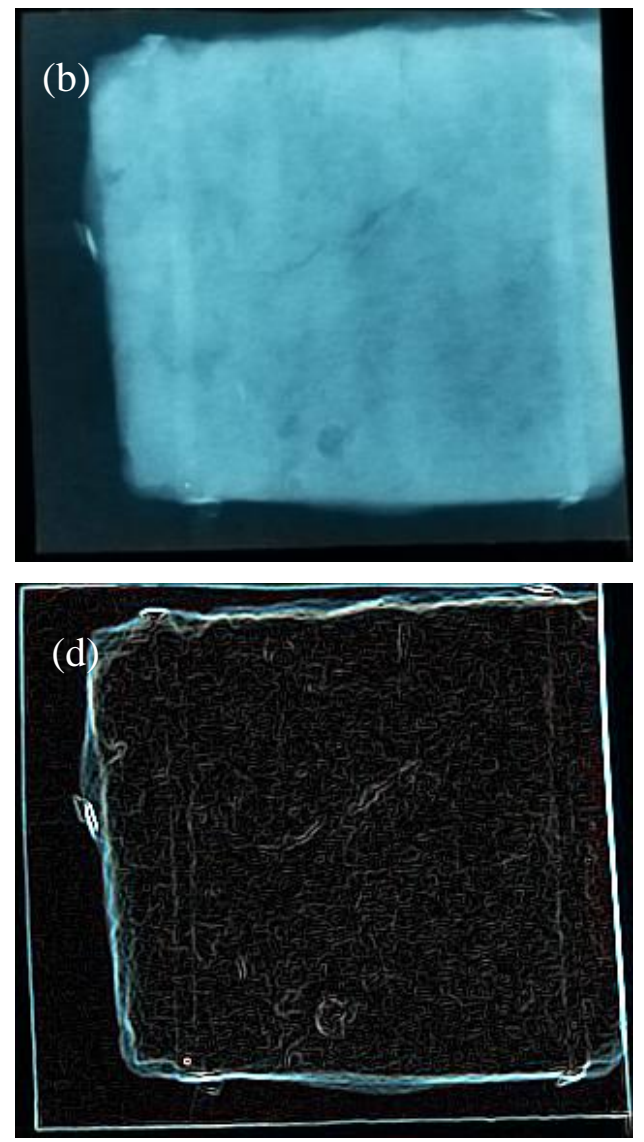

Fig. 16. (a) ) Brick sample from Sonargaon (SB-2); (b) RGB radiograph of SB-2 (319x 286 pixels; RGB; $356 K)$; (c) ROI 8-bit image of SB-2 extracted from RGB using ImageJ (89K); (d) Enhancement of edges of SB-2.

The second brick sample from Sonargaon SB-2, along with its radiographs, ROI and edge profile was shown in Fig. 16. Some dark spots appeared in the radiographic image of SB-2. The unsmooth surface of the sample was clearly visible in Fig. 16 (d). The variation of gray value or the image intensity with respect to a pixel location in the image was plotted in Fig. 17 (a) and a 3-D version of this plot was also shown in Fig. 17 (b). The intensity profile showed a zigzag profile with the maximum intensity of 173.8795 at a pixel position of 243 and a minimum gray value was found 138.6473 at a pixel location of 124 . The percentage of variation in gray value was $20.26 \%$.
Few dark spots have been appeared in the radiographs of all the samples, indicating the vacant place and cracks inside the sample. The radiographic images had been appeared to be inhomogeneous except MB-1 where variation in gray value was lowest among all samples. The dark spots and cracks have been the indications of voids or free spaces and in-built cracks inside the sample through which neutron passed and reached the detector more frequently than other portions of the sample. These spots might have become trigger points to break down the sample. These had been the internal porosities where water is possible to be trapped and these vacant spaces may allow to grow different moss or even the roots of trees from the Moraceae family. 
(a)

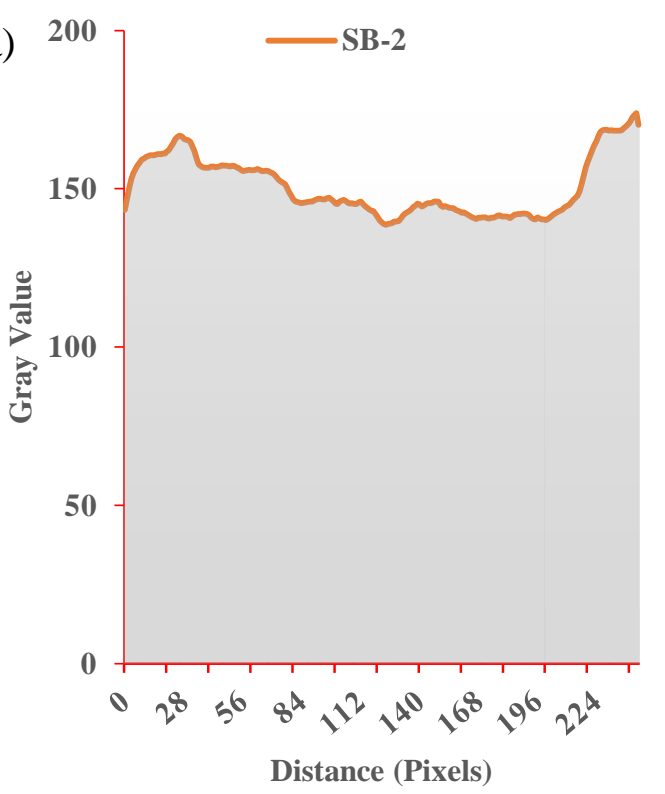

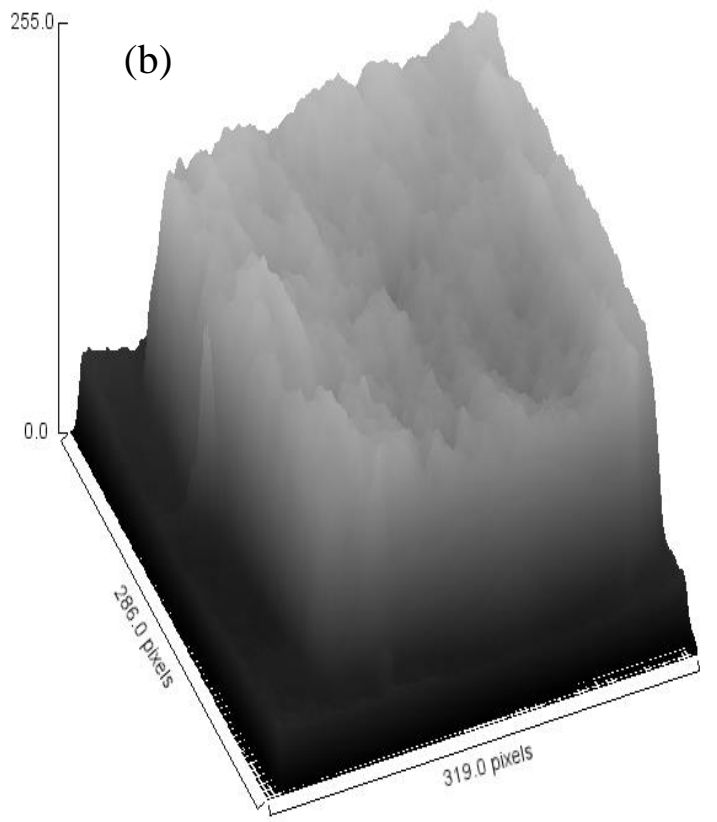

Fig. 17. (a) Image intensity at different pixel distances for ROI of SB-1; (b) Gray value distribution in 3-D surface plot of 8-bit image from $S B-1$.

Gray value comparison curves had been shown in Fig. 18 where four line graphs of gray values resolution corresponding to the homogeneity of that individual samples at dry condition have been depicted using ImageJ software. Variation in homogeneity or structural deformation from edge to edge inside the sample has been implemented through the changes in Y-axis values for these individual curves. Among these four curves, the yellow lined curve corresponding to MB-1 has been seemed to be more consistent showing very few signs of deformation in homogeneity of constituent materials inside the sample except the edge region. Because the corrosion parts of the edge region are not perfectly cut. The other three curves were also nearly flat showing very few signs of deformation.

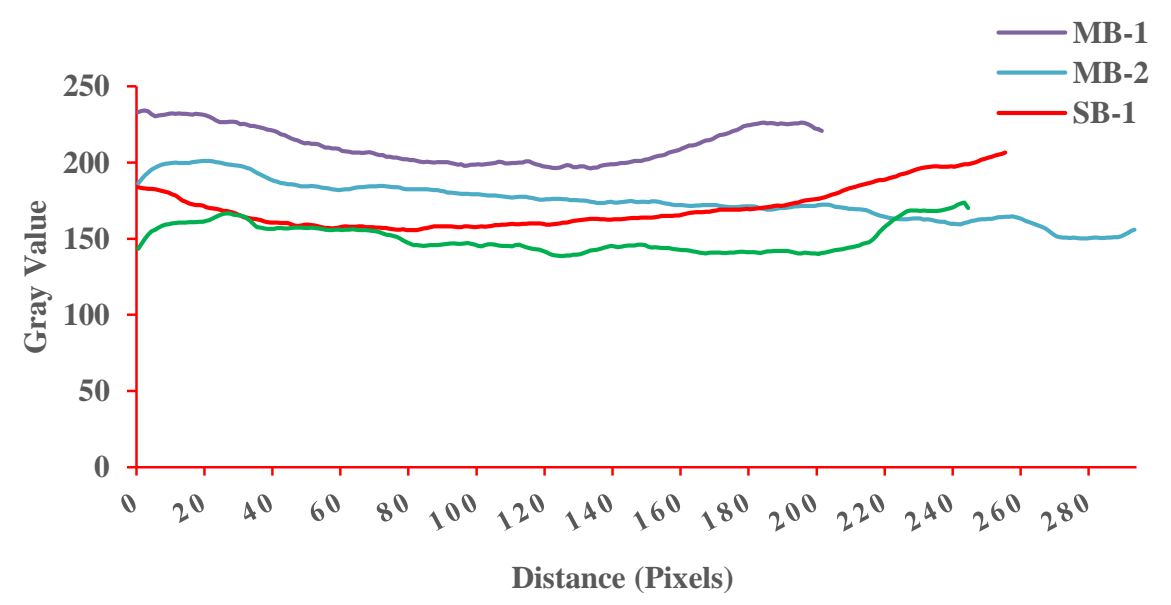

Fig. 18. Comparison of gray values with pixels among different brick samples for ROI.

Fig. 19 illustrated the histogram plots of all samples for ROI. Histogram of an image, like other histograms, also shows frequency. But an image histogram shows the frequency of pixels intensity values. In an image histogram, the $\mathrm{x}$-axis shows the gray level intensities and the $y$ axis shows the frequency of these intensities. 
A histogram measures and illustrates the characteristics of brightness and contrast of a digital image. In the histogram, the $\mathrm{X}$-axis represents the intensity of a given pixel, and $\mathrm{Y}$-axis represents the counts, i.e., the number of the pixels that possess that intensity in the image. As in 8-bit gray scale images in this study, the $\mathrm{X}$-axis in a histogram represents the intensity range 0 through 255. Fig. 23 shows the histogram of MB-1. The neutron images obtained originally from the digital camera are 24-bit RGB color images, which are also referred to as a true color images. It is well known that any color can be represented as a mixture of varying levels of three primary colors of light, i.e., red, green, and blue. Thus, the RGB image in which RGB stands for red, green and blue, respectively is the most straightforward way to represent color images by using those three primary colors of light. In a 24-bit RGB bitmap image, each pixel contains a 24-bit value called RGB triplet made of three separate 8-bit scales. Each scale represents the level of its respective color channel, i.e., red, green or blue. The brightness values represent levels with a 256-level scale, ranging from 0 (black) to 255 (brightest). Before the intensity analysis was performed, those RGB neutron images were transformed into 8-bit gray scale images by using the default procedure provided by the software ImageJ. Afterwards, those 8-bit gray scale images are processed with pseudo-color, which is used to visually amplify specific intensities that are difficult to distinguish from their surroundings by rendering those intensity values in different colors. The process of filtering of pixels is also used in order to choose and distinguish the pixels of interest from their surroundings. In the processed still neutron images, the lower pixel intensity indicates the lower attenuation of neutrons at that location. For instance, in an 8-bit gray scale neutron image of the bricks, the pixel intensity of the area in the edges always has a lower value than that of the area in the center of the bricks.

As it can be seen from the graphs that most of the bars that have high frequency lies in the second half portion which is the brighter portion. That means that the images we have got are brighter. And this can be proved from the image too. Especially in case of MB-1 all the bars lies at the high end of gray values since MB-1 appeared as more brighter images among all, In Fig. 19 (e), all bar lies in the slightly lower region of gray values which indicated that SB-2 is darker image compared to others. Histograms have many uses in image processing. We can predict an image by just looking at its histogram. The histogram is used for brightness purposes, adjusting the contrast of an image and to equalize an image and thresholding. 

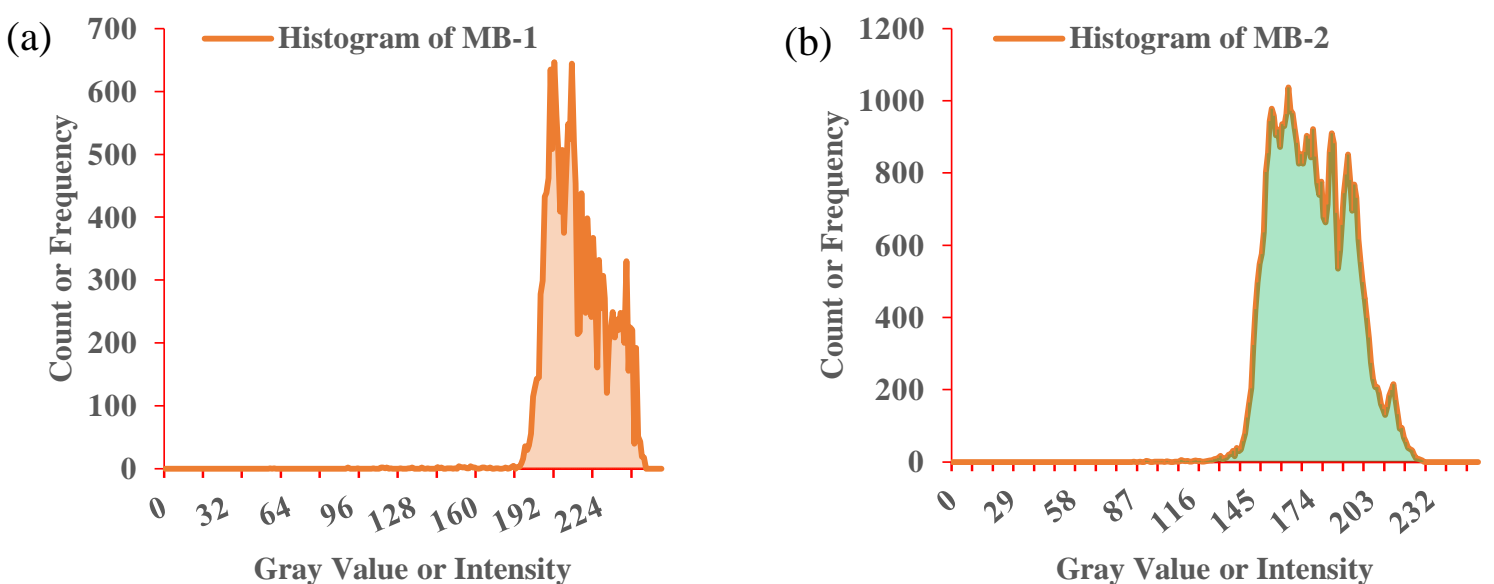

Gray Value or Intensity
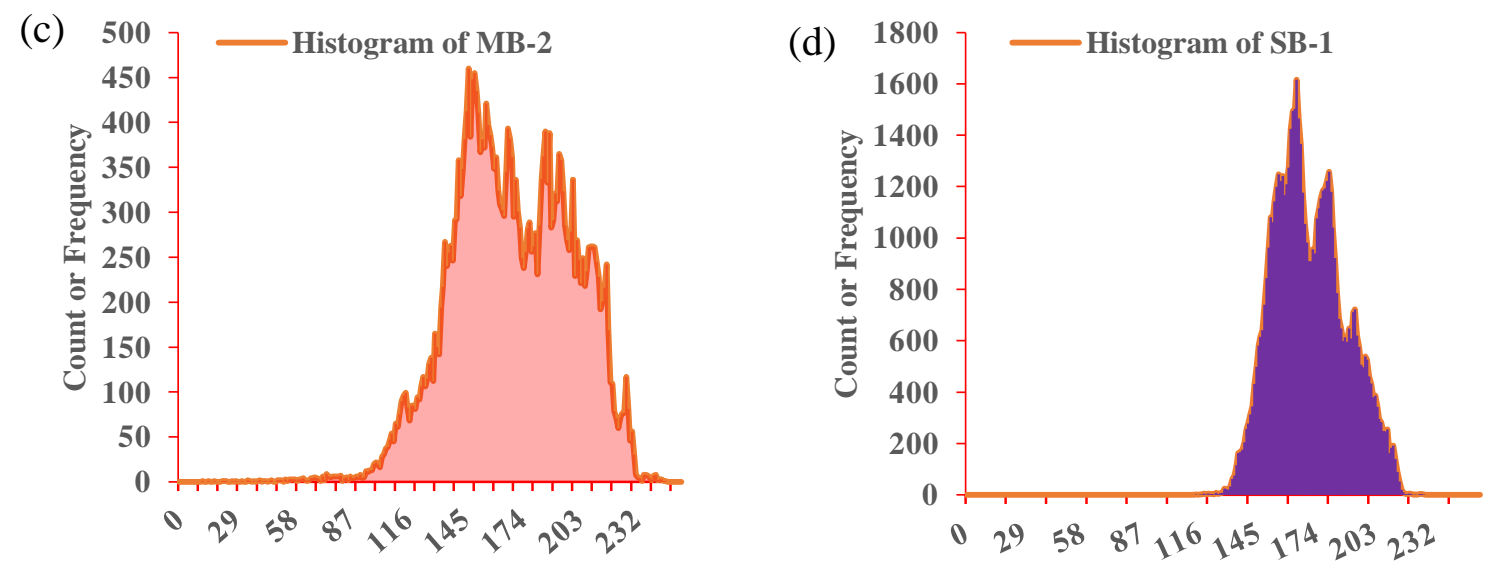

Gray Value or Intensity

Gray Value or Intensity

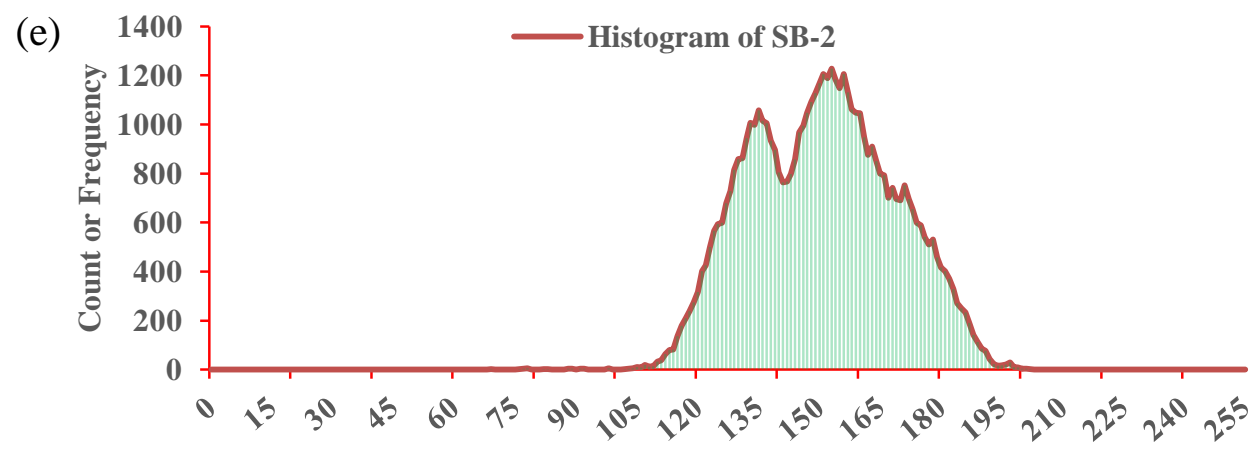

Gray Value or Intensity

Fig. 19. Comparison of gray values with pixels among different brick samples for ROI.

Feature extraction plays a vital role in pattern classification. Gray Level Co-occurrence Matrix (GLCM) features were determined along $0^{\circ}$ for all radiographs. In this work, 9 (nine) texture features shown in Table 1 are extracted from the texture feature sub-space based on GLCM using MATLAB codes. The contrast is a measure of the spatial frequency of an image, i.e. pixel intensity and its neighbor over the image. It also determines the amount of local variations present in the image. The contrast is measured by the difference in the color and brightness of each object with other objects within the same field of view (Mall et al., 2019). Variance present in the radiographs is measured through it. Its value is high in the case of SB2 and low for MB-2 (lateral view) has huge variation in the matrix. 
Table 1. Different texture features extraction for brick sample images

\begin{tabular}{ccccccc}
\hline & Variables & MB-1 & $\begin{array}{c}\text { MB-2 } \\
\text { (Top View })\end{array}$ & $\begin{array}{c}\text { MB-2 } \\
\text { (Lateral View) }\end{array}$ & SB-1 & SB-2 \\
\hline $\begin{array}{c}\text { Contrast } \\
\text { Group }\end{array}$ & Contrast & 0.051506 & 0.042496 & 0.040430 & 0.040955 & 0.058418 \\
& Dissimilarity & 0.051090 & 0.042496 & 0.040430 & 0.040955 & 0.058418 \\
& Homogeneity & 0.974496 & 0.978752 & 0.979785 & 0.979523 & 0.970791 \\
Orderliness & Angular Second & 0.556368 & 0.370108 & 0.264327 & 0.384641 & 0.389248 \\
Group & Moment & & & & \\
& Maximum Probability & 0.717290 & 0.525594 & 0.321970 & 0.531755 & 0.546675 \\
& Entropy & 0.394308 & 0.525271 & 0.674714 & 0.503920 & 0.521027 \\
& Energy & 0.745901 & 0.608365 & 0.514127 & 0.620194 & 0.623897 \\
Statistics Group & Mean & 7.165472 & 5.945452 & 5.787373 & 5.846073 & 5.163969 \\
& Variance & 4.098717 & 5.415515 & 6.325594 & 5.123084 & 3.767700 \\
\hline
\end{tabular}

The dissimilarity feature measures the distance between pairs of objects in the ROI. It measures the gray level mean difference in the distribution of the image. A larger value implies a greater disparity in intensity values among neighboring pixels (Mall et al., 2019). There is a similarity between the contrast and dissimilarity of an image. In all radiographs, values of contrast and dissimilarity are the same except the MB-1 sample.

The homogeneity is commonly referred to as inverse different movement. It measures image homogeneity with larger values for smaller gray tone differences in pair objects. Homogeneity decreases as contrast increases with constant energy (Mall et al., 2019). It is the inverse of contrast weight. Its value ranges from 0 to 1 . Since SB-2 had the highest contrast, it has the lowest homogeneity and MB-2 (lateral view) has the highest homogeneity due to its low contrast. Thus the obtained results conform to the inverse relation between contrast and homogeneity.

Angular Second Moment (ASM) is also known as uniformity or square of energy. It is the sum of squares of entries in the GLCM. Highest ASM was found for MB-1 and the lowest was recorded for MB-2 (lateral view). MB-2 (top view) showed the second lowest value of ASM.

The maximum probability measures the maximum likelihood of producing the pixels of interest. MB-1 has a high value of maximum probability and MB-2 (top view), SB-1 and SB-2 have close values for maximum probability.
Entropy shows the amount of information of the image that is needed for image compression. Entropy measures the loss of information or message in a transmitted signal and also measures the image information (Mohanaiah et al., 2013). Entropy is a measure of randomness; it also describes the distribution variance in a region. MB-1 was found to have the lowest entropy value and MB-2 (lateral view) had the highest entropy value. Entropy is strongly but inversely correlated to energy as images with larger gray levels have high entropy.

The energy feature measures the degree of pixel pair repetitions. It is the measurement of disorder in texture in an image. With highly correlated pixels, the energy value is very large (Mall et al., 2019). A high value of energy was observed for the MB-1 sample and a low value was noticed in the case of the MB-2 sample. Its value ranges from 0 to 1.

The sum average feature measures the mean of the gray level sum distribution of an image. It expresses the relationship between clear and dense areas in radiographs. MB-1 showed the highest mean value while SB-2 had the lowest mean value.

The sum of squares variance measures the dispersion of the gray level sum distribution of the image. This heterogeneity is strongly correlated to a first-order statistical variable, i.e. standard deviation. As the gray level value differs from its mean value, there is an increase in the variance of the image (Mall et al., 2019). The radiograph of SB-2 showed low variance compared to other radiographs. MB-2 sample emerged with the highest variance among all radiographs. 


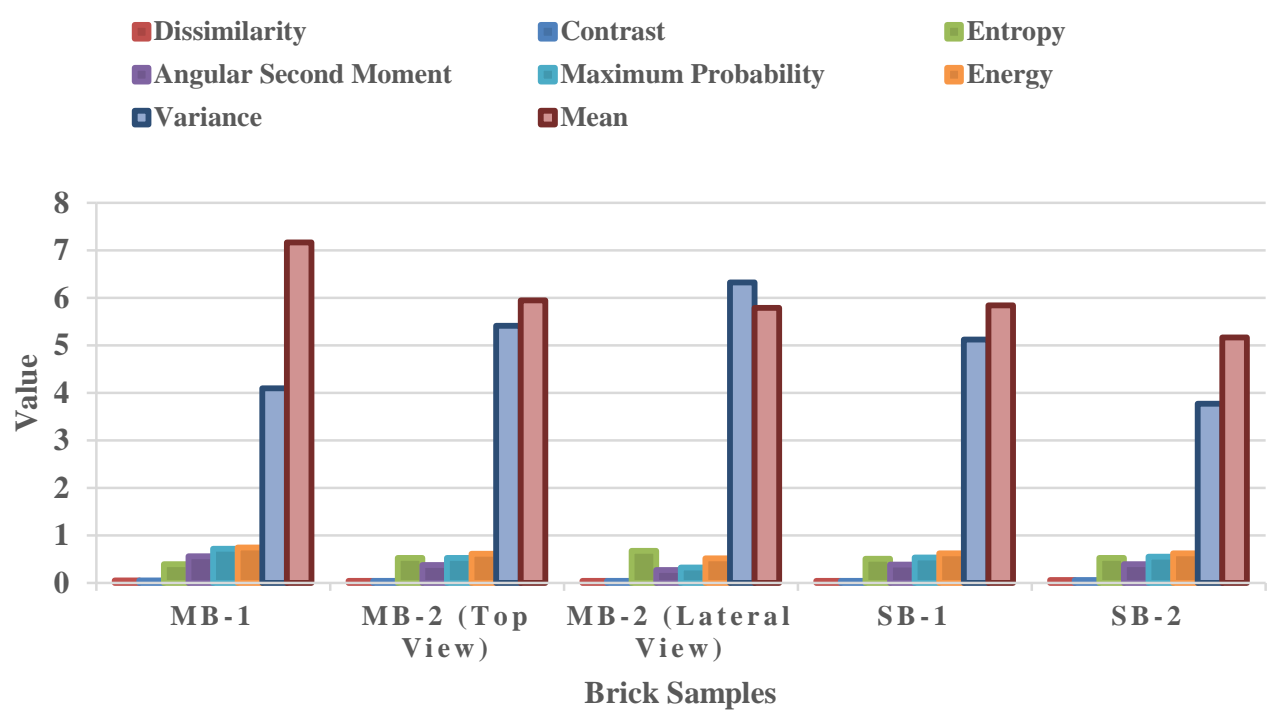

Fig. 20. Variation of extracted texture features of brick samples.

Fig. 20 showed the variation of nine extracted texture features of all the brick samples. The contrast and dissimilarity of all samples are the same and they overlapped on each other. Mean and variance had higher values of greater than 1. SB-2 had the lowest variance whereas MB-2 (lateral view) had the highest variance.

\section{CONCLUSIONS}

The aim of this study is to analyse and process the obtained images using ImageJ and extract texture features by GLCM method as well as illustrate the neutron imaging technique. In this experimental work, the internal structure of the samples has been studied by measuring gray value/intensity distribution, histograms and extracting texture features from the neutron radiographic images of each sample. Variation of gray values of the radiographic images of the samples depicts about uniformity distribution and indications of internal porosity. The images obtained here can tell us about the homogeneity, any voids or cracks etc. The present study has provided some information about the brick manufacturing quality of industries during the ancient time of Bangladesh. These samples are still quite competitive to the samples of the present time. NR is a Non Destructive testing technique and perhaps the only alternative to assess these properties of the samples accurately. This study shows the capability and ability of neutron radiation imaging to obtain information about building media and their structural properties through image processing and texture analysis methods and will enhance the R \& D capabilities through future studies at BTRR. In addition, there will likely be academic interest in the study of historic artifacts including ancient pottery, or in rare fossils for instance from various archaeological sites in Bangladesh.

\section{ACKNOWLEDGMENT}

This work was supported by the Institute of Nuclear Science and Technology (INST) and Center for Research Reactor (CRR) of Bangladesh Atomic Energy Commission (BAEC).

\section{DECLARATION OF COMPETING INTEREST}

The authors declare that they have no known competing financial interests or personal relationships that could have appeared to influence the work reported in this paper.

\section{CREDIT AUTHORSHIP CONTRIBUTION STATEMENT}

Robin Barman: Conceptualization, Methodology, Investigation, Validation, Writing- Original draft. Sudipta Saha: Conceptualization, Formal analysis, Investigation, Samples' Collection and Processing. Md. Sayed Hossain: Formal Analysis, Writing- Review \& Editing, Visualization, Software Analysis. Debasish Chowdhury: Supervision, Writing- Review \& Editing. Anik Das: Writing - Review \& Editing. Md. Kaosar Ahmmad Rabby: Data Curation. Abdullah al Mahmud: Data Curation. 


\section{REFERENCES}

Alazawi SA, Shati NM, Abbas AH (2019). Texture features extraction based on GLCM for face retrieval system. Period Eng Nat Sci 7:1459-1467.

Barman R, Hossain MS, Das A, Rabby MKA. 2021. Investigation of Radiation Shielding Characteristic Features of Different Wood Species. Radiat Phys Chem 109927.

https://doi.org/10.1016/j.radphyschem.2021.109927

Brenizer JS (2013). A review of significant advances in neutron imaging from conception to the present. Phys Procedia 43:10-20.

Chadwick J (1932). Possible existence of a neutron. Nature 129:312.

Chankow N, Punnachaiya S, Wonglee S (2010). Neutron radiography using neutron imaging plate. Appl Radiat Isot 68:662-664.

Das A, Hossain MS, Rabby MKA, et al (2021a). Prospect of Internet Reactor Laboratory in Bangladesh. J Nucl Ene Sci Power Generat Technol 10:2.

Das A, Hossain MS, Rabby MKA, Barman R (2021b). Virtual reactor laboratory integrated with cyber security from Bangladesh perspective. Int J Adv Nucl React Des Technol 3:184-193. https://doi.org/https://doi.org/10.1016/j.jandt.2021.09. 005

Department of Archaeology, Ministry of Cultural Affairs $G$ of the PR of B (2003). Brochure: Mahasthan - the earliest city-site of Bangladesh.

Garbe U, Ahuja Y, Ibrahim R, et al (2017). Industrial application experiments on the neutron imaging instrument DINGO. Phys Procedia 88:13-18.

Hossain MM (2006). Mahasthan: Anecdote to history. Dibyaprakash.

Islam S, Kabir MA (2019). Endangered Historical Sites Conservation in Bangladesh: A Case Study of the Sonargaon. SAMSMRITI - SAMS J 13:30-45.

Kallmann H (1948). Neutron radiography. Research 1:254-260.

Khan MMH (2020). Role of Stakeholders in Heritage Management in Bangladesh: A Case Study of Mahasthangarh. CenRaPS J Soc Sci 2:354-372.
Lehmann E, Trtik P, Ridikas D (2017). Status and perspectives of neutron imaging facilities. Phys Procedia 88:140-147.

Lehmann EH, Ridikas D (2015). Status of Neutron Imaging-Activities in a Worldwide Context. Phys Procedia 69:10-17.

Luo X (2007). Study on infrastructure materials using neutron radiography and diffraction

Mall PK, Singh PK, Yadav D (2019). GLCM based feature extraction and medical X-RAY image classification using machine learning techniques. In: 2019 IEEE Conference on Information and Communication Technology. IEEE, pp 1-6.

Mannes D, Schmid F, Frey J, et al (2015). Combined Neutron and $\mathrm{X}$-ray imaging for non-invasive investigations of cultural heritage objects. Phys Procedia 69:653-660.

Mohanaiah P, Sathyanarayana P, GuruKumar L (2013). Image texture feature extraction using GLCM approach. Int J Sci Res Publ 3:1-5.

Orina I, Manley M, Williams PJ (2017). Non-destructive techniques for the detection of fungal infection in cereal grains. Food Res Int 100:74-86.

Oswald SE, Menon M, Carminati A, et al (2008). Quantitative imaging of infiltration, root growth, and root water uptake via neutron radiography. Vadose Zo J 7:1035-1047.

Öztürk Ş, Akdemir B (2018). Application of feature extraction and classification methods for histopathological image using GLCM, LBP, LBGLCM, GLRLM and SFTA. Procedia Comput Sci 132:40-46.

Sharmin N (2019). Cultural significance assessment, Panamnagar: a testimony of historic Bengal.

Thewlis J (1956). Neutron radiography. Br J Appl Phys $7: 345-350$ https://doi.org/10.1088/05083443/7/10/301

Zhang P, Wittmann FH, Lura P, et al (2018). Application of neutron imaging to investigate fundamental aspects of durability of cement-based materials: A review. Cem Concr Res 108:152-166. 